\title{
Multiuser 3D massive MIMO transmission in full-duplex cellular system
}

\author{
Dongmei Zhang ${ }^{1}$, Ximing Wang ${ }^{1 *} \mathbb{C}$, Kui Xu$^{1}$, Yijun Yang ${ }^{2}$ and Wei Xie ${ }^{1}$
}

\begin{abstract}
In this paper, we provide an effective multiuser transmission scheme in three-dimensional (3D) massive multiple-input multiple-output (MIMO) cellular systems, where the full-duplex (FD) base station (BS) equips two separate large-scale uniform planar antenna array (UPA). In order to reduce the computational and implementation complexity, we investigate the characteristic of the beam-domain (BD) 3D massive MIMO channels and self-interference (SI) channel. We propose the 3D multiuser beamspace transmission (MUBT) scheme that requires the spatial angular information of the users and the SI channel. We show that, due to the reduced dimension property of the effective beamspace channel, the overhead for channel estimation is reduced. Furthermore, a user scheduling algorithm is proposed to enable the 3D MUBT scheme in the FD systems. Finally, both the theoretical analysis and simulation results show the the SI can be effectively reduced and demonstrate the effectiveness and superiority of the proposed 3D MUBT scheme on spectral efficiency compared with the conventional half-duplex (HD) and FD transmission schemes.
\end{abstract}

Keywords: Multiuser cellular system, 3D massive MIMO, Full-duplex, Beamspace channel representation, Minimum mean square error (MMSE) channel estimation, Beamforming

\section{Introduction}

With the booming development of smart terminals and the growing demand for new mobile services, the mobile Internet traffic will continue to grow exponentially, which will increase by roughly 1000 times beyond 2020 . Multiple-input multiple-output (MIMO) techniques can obtain multiplexing gain, diversity gain, and antenna gain by exploiting the spatial dimension of wireless resources, which as a result can significantly enhance the capacity and reliability in wireless communications [1]. In order to further improve the spectral efficiency (SE) of the communication systems, massive MIMO has attracted considerable attention. Massive MIMO is first advocated in [2], which can make the simple linear precoder tend to optimal and eliminate the noise along with uncorrelated interference by simply scaling up the numbers of antenna elements at the base station (BS). In this way, the SE of massive MIMO systems is improved dramatically because more users can be served in the same timefrequency resource. Furthermore, massive MIMO enables

\footnotetext{
*Correspondence: Igdxwxm@sina.com

${ }^{1}$ Army Engineering University of PLA, No. 88, Houbiaoying Street, Nanjing,

China

Full list of author information is available at the end of the article
}

users to reduce the transmit power arbitrarily without compromising the SE [3].

However, in the practical massive MIMO systems, all these performance gains are profitted from the channel state information (CSI) that is available. Time-division duplex (TDD) seems to be more suitable for massive MIMO systems since the channel reciprocity can be exploited to obtain the instantaneous downlink (DL) CSI through uplink (UL) training [2]; thus, the overhead for channel estimation is linear with the number of user antennas. For this reason, a great deal of research work has been done for TDD [3-7]. However, the imperfect calibration between the UL/DL radio frequency chains [8] and pilot contamination $[9,10]$ in the practical TDD systems limits the performance gains of massive MIMO, which as a result motivates the research on frequency-division duplex (FDD) massive MIMO systems [11-13]. Since the channel reciprocity does not hold for FDD systems, the training overhead for DL estimation scales linearly with the number of BS antennas. This poses a heavy burden on user equipments and the feedback links in the system where the BS is equipped with a large number of antennas. One attempt is to adopt the closed-loop training scenarios to sequentially design the optimal beam patterns 
[11]. Another alternative method is to exploit the lowrank property of channel covariance matrix in massive MIMO systems [12, 13]. Utilizing the correlation between channels, the reduced dimension effective channels can be obtained by the eigen-decomposition of channel covariance matrix, which can reduce the overhead of training and feedback.

Furthermore, most of the prior works assume the largescale antenna array deployed along the horizontal axis, which is not applicable for most BSs due to the limited space on the roof or mast. Three-dimensional (3D) massive MIMO, which is referred to as full-dimension MIMO, can overcome this practical challenge because large-scale antennas are arranged in both the horizontal and vertical dimensions when two-dimensional (2D) uniform planar antenna array (UPA) are deployed. In this way, the extra degrees of freedom for vertical dimension can be exploited to improve the capability of serving 3D distributed users, as illustrated in Fig. 1. In [14], the channel correlation matrix based on a generic raytracing 3D channel model is investigated, and the study shows that the channel correlation matrix can be well approximated by the Kronecker production of the correlations in horizontal and vertical directions. References $[12,15-17]$ investigate the DL transmission for 3D massive MIMO systems. Based on both statistical and instantaneous CSI, [12] proposes a joint spatial division and multiplexing with $3 \mathrm{D}$ precoding scheme. By exploiting the Kronecker structure of the 3D MIMO channel matrix, Wang et al. [15] propose a $2 \mathrm{D}$ precoding scheme to fully exploit the degrees of freedom provided by the vertical dimension, which can reduce the multiuser interference (MUI) and inter-cell interference (ICI). Li et al. $[16,17]$ assumes that the BS has only the statistical CSI of each user. Through eigen-decomposition of horizontal and vertical correlation matrices, the beamforming vector for each user are obtained. Moreover, the space division multiple access transmission scheme based on the 3D beamforming is proposed.

Both TDD and FDD systems are half-duplex (HD) systems that assign orthogonal time or frequency resources to the UL and DL, which theoretically leads to half of the time-frequency resources wasted. With the development of signal and hardware processing techniques, recently, the full-duplex (FD) techniques become one of the attractive options for wireless communications. The severe self-interference (SI) due to the signal leakage from the transmitter to the receiver is one of the major challenges for FD communications, which results in the rapid development of various kinds of SI cancellation (SIC) techniques [18-20]. Due to the FD transmission, the DL users may suffer from the interference transmitted by UL users in cellular systems, which can be controlled by using different beams [21-23]. In order to achieve higher SE performance, a lot of research has been done on FD massive MIMO technology [24-28]. Xia et al. [28] shows that adopting linear beamforming techniques can significantly reduce the adverse impact of SI, and FD massive MIMO systems outperform the HD counterpart in SE even without active SI cancellation.

In this paper, we assume that two separate large-scale UPAs are deployed at the BS, and investigate 3D multiuser transmission in the FD massive MIMO cellular system. The contributions of our works are as follows:

- The beam-domain (BD) characteristic of the 3D channel is investigated, including UL, DL, and SI channels. In the BD channel, most of the power is

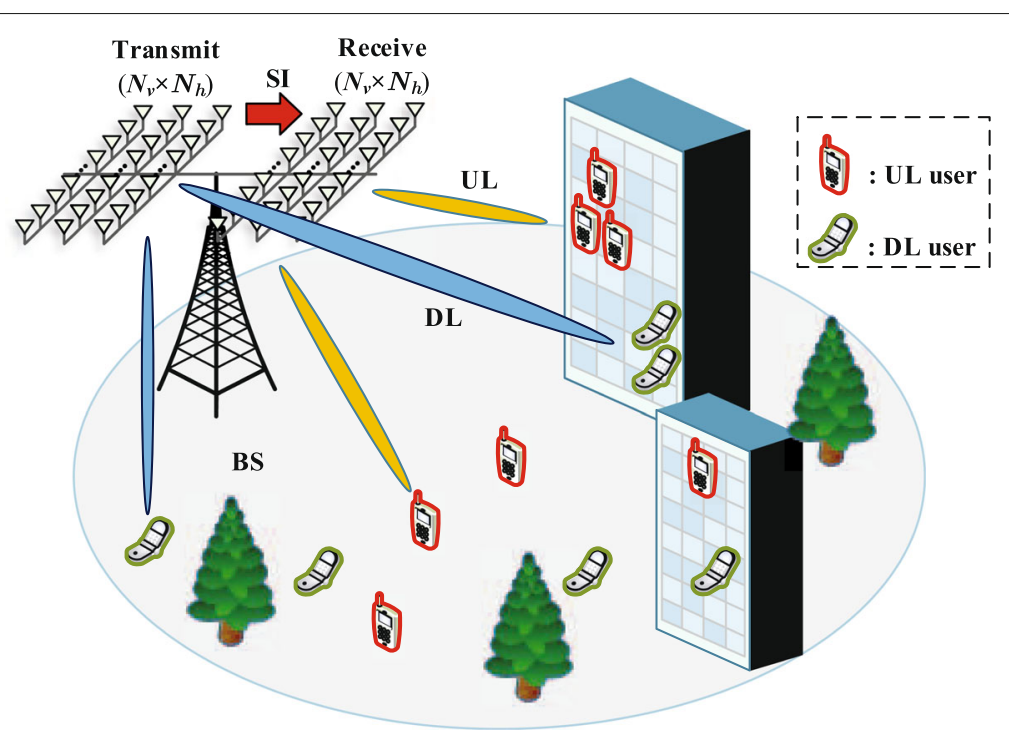

Fig. 1 Single-cell multiuser 3D massive MIMO system 
concentrated on each user's own beamspace. The algorithm of calculating each user's best beamspace is provided.

- By exploiting the low-dimension property of the effective beamspace channels in the $\mathrm{BD}$, we propose the 3D multiuser beamspace transmission (MUBT) scheme which processes the signals in each users's beamspaces. Utilizing the orthogonality of beamspaces between groups, we show that our the 3D MUBT can eliminate the inter-group interference (IGI), and significantly reduce the overhead of channels estimation.

- The SI channel in 3D massive MIMO is modeled, and the beamspaces of the SI channel are derived. Based on the beamspaces of SI channel and features of co-channel interference (CCI) within the cellular systems, we introduce a simple cell partitioning strategy and propose a user-scheduling algorithm. Both the theoretical analysis and simulation results show that the proposed scheme reduce the SI effectively.

Notation: We use boldface uppercase letter $\mathbf{A}$ and boldface lowercase letter a to represent matrix and column vector, respectively. $\mathbb{E}(\cdot),\|\cdot\|,(\cdot)^{*},(\cdot)^{H}, \Phi(\cdot)$ and $\operatorname{Tr}(\cdot)$ stand for the expectation, the Euclidean norm, the conjugate, the conjugate transpose, the spectral radius of a matrix and trace of a matrix, respectively. $|\mathcal{B}|$ denotes the cardinality of a set $\mathcal{B} .[\mathbf{A}]_{i, j}$ denotes the $i$ th-row and $j$ thcolumn entry of matrix $\mathbf{A}$. $\mathbf{I}_{N}$ represents the $N \times N$ identity matrix. $\mathcal{C} N\left(\mu, \sigma^{2}\right)$ stands for the complex-Gaussian distribution with mean of $\mu$ and variance of $\sigma^{2}$. $\mathbf{e}_{i}$ represents a vector whose $i$ th entry is 1 and the other are $0 . \delta(\cdot)$ is the Dirac delta function.

\section{Methods}

The outline of this paper is as follows: Section 3 describes the system and the channels model. In Section 4, the BD characteristics of 3D massive MIMO channels are investigated and the beamspace representation of channel is given. In Section 5, we detail the proposed 3D MUBT scheme, which includes user scheduling, channel estimation, and data transmission. In Section 6, we perform various simulation results using MATLAB to demonstrate the effectiveness of the proposed scheme, and furthermore, the existing approaches are compared under the same setting to show the superiority of the proposed scheme. Finally, the concluding remarks are offered in Section 7.

\section{System and channel models}

As shown in Fig. 1, we consider a 3D multiuser massive MIMO system, where $K_{u}$ UL users and $K_{d}$ DL users are distributed in 3D space. We assume that all the users work in HD mode and equipped with single antenna. The FD
BS deploys two separate large-scale UPAs ${ }^{1}$ (one for transmitting and one for receiving), and both of them has $N_{v}$ antennas in the vertical dimension and $N_{h}$ antennas in the horizontal dimension and each dimension is a uniform linear antenna array (ULA). Let $d_{\nu}$ and $d_{h}$ denote the distance of adjacent antennas in the vertical and horizontal directions.

\subsection{UL and DL channel models}

In this paper, we consider a 3D massive MIMO channel model and assume that the channels between the BS and users passes through a great number of rays [29]. Therefore, the channel of UL user $k_{u}$ can be written as:

$$
\mathbf{G}_{k_{u}}=\iint_{\left(\theta_{k_{u}}, \varphi_{k_{u}}\right) \in \Delta_{k_{u}}} r_{u}\left(\theta_{k_{u}}, \varphi_{k_{u}}\right) \mathbf{A}\left(\theta_{k_{u}}, \varphi_{k_{u}}\right) d \theta_{k_{u}} d \varphi_{k_{u}}
$$

where $\theta_{k_{u}}$ and $\varphi_{k_{u}}$ represent the direction of arrival (DoA) of user $k_{u}$ in vertical and horizontal directions; $\Delta_{k_{u}}=$ $\left\{\left(\theta_{k_{u}}, \varphi_{k_{u}}\right) \mid \theta_{k_{u}} \in\left[\theta_{k_{u}}^{\min }, \theta_{k_{u}}^{\max }\right], \varphi_{k_{u}} \in\left[\varphi_{k_{u}}^{\min }, \varphi_{k_{u}}^{\max }\right]\right\}$ denote the angular spread (AS) range resulting from scatters in the vertical and horizontal directions; and $r_{u}\left(\theta_{k_{u}}, \varphi_{k_{u}}\right)$ denotes the UL complex response gain. Here, $\mathbf{A}\left(\theta_{k_{u}}, \varphi_{k_{u}}\right) \in \mathbb{C}^{N_{v} \times N_{h}}$ is the array response matrix which can be expressed as:

$$
\begin{aligned}
& \mathbf{A}\left(\theta_{k_{u}}, \varphi_{k_{u}}\right)= \\
& {\left[\begin{array}{ccc}
1 & \cdots & e^{j\left(N_{h}-1\right) h_{k_{u}}} \\
e^{j v_{k_{u}}} & \cdots & e^{j\left[v_{k_{u}}+\left(N_{h}-1\right) h_{k_{u}}\right]} \\
\vdots & \ddots & \vdots \\
e^{j\left(N_{v}-1\right) v_{k_{u}}} & \cdots & e^{j\left[\left(N_{v}-1\right) v_{k_{u}}+\left(N_{h}-1\right) h_{k_{u}}\right]}
\end{array}\right],}
\end{aligned}
$$

where $v_{k_{u}}=\frac{2 \pi d_{v}}{\lambda} \cos \theta_{k_{u}}$ and $h_{k_{u}}=\frac{2 \pi d_{h}}{\lambda} \sin \theta_{k_{u}} \cos \varphi_{k_{u}}$, and $\lambda$ is the carrier wavelength. Define

$$
\begin{aligned}
& \mathbf{a}\left(v_{k_{u}}\right)=\left[1, e^{j v_{k_{u}}}, \cdots, e^{j\left(N_{v}-1\right) v_{k_{u}}}\right]^{T}, \\
& \mathbf{b}\left(h_{k_{u}}\right)=\left[1, e^{\left.j h_{k_{u}}, \ldots, e^{j\left(N_{h}-1\right) h_{k_{u}}}\right]^{T},}\right.
\end{aligned}
$$

then we can rewrite $\mathbf{A}\left(\theta_{k_{u}}, \varphi_{k_{u}}\right)=\mathbf{a}\left(v_{k_{u}}\right) \mathbf{b}\left(h_{k_{u}}\right)^{T}$. As a result, the UL channel matrix can be rewritten as:

$$
\mathbf{G}_{k_{u}}=\iint_{\left(\theta_{k_{u}}, \varphi_{k_{u}}\right) \in \Delta_{k_{u}}} r_{u}\left(\theta_{k_{u}}, \varphi_{k_{u}}\right) \mathbf{a}\left(v_{k_{u}}\right) \mathbf{b}\left(h_{k_{u}}\right)^{T} d \theta_{k_{u}} d \varphi_{k_{u}} .
$$

Therefore, the equivalent vectorial form of $\mathbf{G}_{k_{u}}$ is:

$$
\begin{aligned}
& \mathbf{g}_{k_{u}}=\operatorname{vec}\left(\mathbf{G}_{k_{u}}\right)= \\
& \quad \iint_{\left(\theta_{k_{u}}, \varphi_{k_{u}}\right) \in \Delta_{k_{u}}} r_{u}\left(\theta_{k_{u}}, \varphi_{k_{u}}\right) \mathbf{b}\left(h_{k_{u}}\right) \otimes \mathbf{a}\left(v_{k_{u}}\right) d \theta_{k_{u}} d \varphi_{k_{u}} .
\end{aligned}
$$


Similarly, define $\theta_{k_{d}}$ and $\varphi_{k_{d}}$ as the direction of departure (DoD) of DL user $k_{d}$ in vertical and horizontal dimension, and let $\Delta_{k_{d}}=$ $\left\{\left(\theta_{k_{d}}, \varphi_{k_{d}}\right) \mid \theta_{k_{d}} \in\left[\theta_{k_{d}}^{\min }, \theta_{k_{d}}^{\max }\right], \varphi_{k_{d}} \in\left[\varphi_{k_{d}}^{\min }, \varphi_{k_{d}}^{\max }\right]\right\} \quad$ and $r_{d}\left(\theta_{k_{d}}, \varphi_{k_{d}}\right)$ be the AS range of DoD and DL response gain; then, the channel vector of user $k_{d}$ can be expressed as:

$$
\mathbf{g}_{k_{d}}=\iint_{\left(\theta_{k_{d}}, \varphi_{k_{d}}\right) \in \Delta_{k_{d}}} r_{d}\left(\theta_{k_{d}}, \varphi_{k_{d}}\right) \mathbf{b}\left(h_{k_{d}}\right) \otimes \mathbf{a}\left(v_{k_{d}}\right) d \theta_{k_{d}} d \varphi_{k_{d}} .
$$

In this paper, the AS range $\Delta_{k_{u}}$ and $\Delta_{k_{d}}$ are assumed to be relatively narrow, which is reasonable when the BS is set at a relatively high altitude [13].

\subsection{D SI channel model}

We assume that some SIC techniques have been adopted at the BS, e.g., passive SI cancellation technique, so that there exists no light-of-sight (LOS) signals from transmit antenna array to receive antenna array and the two arrays are independent. Moreover, we assume that there are several scatters (e.g., tall buildings) surrounding the arrays; then, the SI signal consists of several non-ligth-of-sight (NLOS) rays.

Let $\mathbf{G}_{S I} \in C^{N_{v} N_{h} \times N_{v} N_{h}}$ be the channel from transmit antenna array to receive antenna array, i.e., SI channel. According to the Kronecker stochastic channel model $[14,30]$, the SI channel can be modeled as

$$
\mathbf{G}_{S I}=\mathbf{C}_{R}^{\frac{1}{2}} \mathbf{G}_{w}\left(\mathbf{C}_{T}^{\frac{1}{2}}\right)^{T}
$$

where $\mathbf{G}_{w}$ is a $N_{v} N_{h} \times N_{v} N_{h}$ random matrix with independent and identical distributed (i.i.d.) $\mathcal{C} N\left(0, \beta_{S I}\right)$ elements. Here, $\beta_{S I}$ can be understood as the level of the residual SI, which is related to the path loss of the SI channel and the capability of the SIC techniques. $\mathbf{C}_{R}$ and $\mathbf{C}_{T}$ denote the receive and transmit spatial correlation matrices which are also the corresponding covariance matrices. In this paper, we assume that only the antenna-domain (passive) SIC techniques are adopted and no analog or digital-domain (active) SIC techniques are used [19].

Now, we need to find out the expressions for every entry of $\mathbf{C}_{R}$ and $\mathbf{C}_{T}$, which are expressed as $\mathbf{C}_{a}, a \in\{R, T\}$ for simplicity of illustration. Define the correlation between $(k, l)$-th and $(p, q)$-th antenna element of antenna array as $\mathbf{C}_{a}^{(k, l),(p, q)}$. According to [14], $\mathbf{C}_{a}^{(k, l),(p, q)}$ can be approximately written as a product of vertical and horizontal correlations; then, the correlation matrix can be expressed as

$$
\mathbf{C}_{a}=\mathbf{C}_{a h} \otimes \mathbf{C}_{a v}
$$

where $\mathbf{C}_{a v}$ and $\mathbf{C}_{a h}$ are the vertical and horizontal correlation with

$$
\begin{aligned}
{\left[\mathbf{C}_{a h}\right]_{l, q} } & =\frac{1}{\sqrt{A}} e^{-\frac{B^{2} \cos ^{2} \varphi_{a}}{2 A}} e^{j \frac{C \cos \varphi_{a}}{A}} e^{-\frac{\left(C \sin \varphi_{a} \sigma a\right)^{2}}{2 A}} \\
{\left[\mathbf{C}_{a v}\right]_{k, p} } & =e^{j \frac{2 \pi d_{v}}{\lambda}(p-k) \cos \theta_{a}} e^{-\frac{1}{2}\left(\xi_{a} \frac{2 \pi d_{v}}{\lambda}\right)^{2}(p-k)^{2} \sin ^{2} \theta_{a}}
\end{aligned}
$$

where $A=\left(\xi_{a} \frac{2 \pi d_{h}}{\lambda}(q-l) \cos \theta_{a}\right)^{2}\left(\sin \varphi_{a} \sigma_{a}\right)^{2}+1, B=$ $\xi_{a} \frac{2 \pi d_{h}}{\lambda}(q-l) \cos \theta_{a}$ and $C=\frac{2 \pi d_{h}}{\lambda}(q-l) \sin \theta_{a}$. Here, $\theta_{a}$, $\varphi_{a}, \xi_{a}$, and $\sigma_{a}$ denote the $\operatorname{DoA}(a=R)$ or $\operatorname{DoD}(a=T)$ in vertical and horizontal directions and the normal distribution variance of the angular perturbation in vertical and horizontal directions. Note that, from (9), we can see that $\mathbf{C}_{a}$ is Hermitian matrix.

\section{Beam-domain characteristics of 3D massive MIMO channel}

In the cellular systems, due to the fact that the antenna array at the BS are generally set at a relative high building where the scatters around are relatively sparse, therefore, most of the energy of the channels concentrate on several spatial directions [31], i.e., several beams of signals. Hence, in order to realize multiuser transmission in the 3D massive MIMO system, we first investigate the physical beam characteristic of 3D massive MIMO channels.

\subsection{Beamspaces of UL and DL channels}

To get started, we introduce the following lemma:

Lemma 1 The channels of UL user $k_{u}$ and $i_{u}$ or DL user $k_{d}$ and $i_{d}(k \neq i)$ are asymptotically orthogonal if their vertical or horizontal AS ranges are disjoint, i.e.,

$$
\begin{aligned}
& \lim _{N_{v}, N_{h} \rightarrow \infty} \frac{1}{N_{v} N_{h}} \operatorname{vec}\left(\mathbf{G}_{k_{x}}\right)^{H} \operatorname{vec}\left(\mathbf{G}_{i_{x}}\right)=0 \Leftrightarrow \\
& \left(\left[\theta_{k_{x}}^{\min }, \theta_{k_{x}}^{\max }\right] \cap\left[\theta_{i_{x}}^{\min }, \theta_{i_{x}}^{\max }\right]\right) \cap\left(\left[\varphi_{k_{x}}^{\min }, \varphi_{k_{x}}^{\max }\right] \cap\left[\varphi_{i_{x}}^{\min }, \varphi_{i_{x}}^{\max }\right]\right)=\varnothing
\end{aligned}
$$

where $x \in\{u, d\}$ represents $U L$ and $D L$, respectively.

Proof Using the property of vec $(\mathbf{A B C})=\left(\mathbf{C}^{T} \otimes \mathbf{A}\right) \operatorname{vec}(\mathbf{B})$ and

$$
\begin{aligned}
\lim _{N_{v}, N_{h} \rightarrow \infty} & =\frac{1}{N_{v} N_{h}}\left[\mathbf{b}\left(h_{k_{x}}\right) \otimes \mathbf{a}\left(v_{k_{x}}\right)\right]^{H}\left[\mathbf{b}\left(h_{i_{x}}\right) \otimes \mathbf{a}\left(v_{i_{x}}\right)\right] \\
& =\frac{1}{N_{v} N_{h}}\left[\mathbf{b}\left(h_{k_{x}}\right)^{H} \mathbf{b}\left(h_{i_{x}}\right)\right] \otimes\left[\mathbf{a}\left(v_{k_{x}}\right)^{H} \mathbf{a}\left(v_{i_{x}}\right)\right] \\
& =\delta\left(h_{k_{x}}-h_{i_{x}}\right) \cdot \delta\left(v_{k_{x}}-v_{i_{x}}\right)
\end{aligned}
$$

Lemma 1 can be easily obtained.

From Lemma 1, we can see that in 3D massive MIMO systems, both the vertical and horizontal resolution are 
high so that users have different DoD or DoA can be simultaneously scheduled, e.g., users in the same building having the same horizontal angle while different in the vertical dimension. Note that, in $2 \mathrm{D}$ massive MIMO system where ULA is deployed at the BS, users can only be distinguished in horizontal plane.

To investigate the physical beam property of 3D massive MIMO, we first define $N_{v} \times N_{v}$ and $N_{h} \times N_{h}$ normalized DFT matrices $\mathbf{F}_{N_{v}}$ and $\mathbf{F}_{N_{h}}$ whose $(i, k)$-th entry is $\left[\mathbf{F}_{x}\right]_{i, k}=\frac{1}{\sqrt{x}} e^{-j \frac{2 \pi}{x} i k}, x \in\left\{N_{v}, N_{h}\right\}$, and introduce the following lemma:

Lemma 2 Define $\tilde{\mathbf{G}}_{k_{x}}=\mathbf{F}_{N_{v}} \mathbf{G}_{k_{x}} \mathbf{F}_{N_{h}}(x \in\{u, d\})$ as the $B D$ channel, then the entries of $\tilde{\mathbf{G}}_{k_{x}}$ have non-negligible values only at limited $2 D$ points as $N_{v}, N_{h} \rightarrow \infty$ which constitutes the beamspace of channel $\mathbf{G}_{k_{x}}$.

Proof We first assume that the UL or DL channels only consist of single ray, i.e., $\mathbf{G}_{k_{x}}=r_{x}\left(\theta_{k_{x}}, \varphi_{k_{x}}\right) \mathbf{A}\left(\theta_{k_{x}}, \varphi_{k_{x}}\right)=$ $r_{k_{x}} \mathbf{a}\left(v_{k_{x}}\right) \mathbf{b}\left(h_{k_{x}}\right)^{T}$, then the $(p, q)$-th entry of $\tilde{\mathbf{G}}_{k_{x}}$ is

$$
\begin{aligned}
{\left[\tilde{\mathbf{G}}_{k_{x}}\right]_{p, q} } & =\frac{r_{k_{x}}}{\sqrt{N_{v} N_{h}}} \sum_{m=0}^{N_{v}-1} e^{-j\left(\frac{2 \pi}{N_{v}} p m-\frac{2 \pi d_{v}}{\lambda} m \cos \theta_{k_{x}}\right)} \\
& \times \sum_{n=0}^{N_{h}-1} e^{-j\left(\frac{2 \pi}{N_{h}} q n-\frac{2 \pi d_{h}}{\lambda} n \sin \theta_{k_{x}} \cos \varphi_{k_{x}}\right)} \\
& =\frac{r_{k_{x}}}{\sqrt{N_{\nu} N_{h}}} e^{-j \frac{N_{\nu}-1}{2} \alpha_{k_{x}}} \frac{\sin \left(N_{\nu} \alpha_{k_{x}} / 2\right)}{\sin \left(\alpha_{k_{x}} / 2\right)} \\
& \times e^{-j \frac{N_{h}-1}{2} \beta_{k_{x}}} \frac{\sin \left(N_{h} \beta_{k_{x}} / 2\right)}{\sin \left(\beta_{k_{x}} / 2\right)}
\end{aligned}
$$

where $\alpha_{k_{x}}=\frac{2 \pi}{N_{v}} p-\frac{2 \pi d_{v}}{\lambda} \cos \theta_{k_{x}}$ and $\beta_{k_{x}}=\frac{2 \pi}{N_{h}} q-$ $\frac{2 \pi d_{h}}{\lambda} \sin \theta_{k_{x}} \cos \varphi_{k_{x}}$. Thus, as $N_{v}, N_{h} \rightarrow \infty$, we can obtain:

$$
\begin{aligned}
& \lim _{N_{v}, N_{h} \rightarrow \infty}\left|\left[\tilde{\mathbf{G}}_{k_{x}}\right]_{p, q}\right|=\sqrt{N_{v} N_{h}}\left|r_{k_{x}}\right| \\
& \times \delta\left(\frac{p}{N_{v}}-\frac{d_{v}}{\lambda} \cos \theta_{k_{x}}\right) \delta\left(\frac{q}{N_{h}}-\frac{d_{h}}{\lambda} \sin \theta_{k_{x}} \cos \varphi_{k_{x}}\right) .
\end{aligned}
$$

Apparently, for any DoA or $\operatorname{DoD}\left(\theta_{k_{x}}, \varphi_{k_{x}}\right)$, there is a $\left(p_{0}, q_{0}\right)$ to satisfy (13) nonzero, i.e., $p_{0}=N_{v} \frac{d_{v}}{\lambda} \cos \theta_{k_{x}}$ and $q_{0}=N_{h} \frac{d_{h}}{\lambda} \sin \theta_{k_{x}} \cos \varphi_{k_{x}}$. Hence, the point $\left(p_{0}, q_{0}\right)$ indicates the direction of the signal, i.e., $\theta_{k_{x}}=\arccos \frac{p_{0} \lambda}{d_{v} N_{v}}$ and $\varphi_{k_{x}}=\arccos \frac{q_{0} \lambda}{d_{h} N_{h} \sin \theta_{k_{x}}}$. Particularly, $p_{0}$ and $q_{0}$ are usually not integers, which as a result will cause the power at point $\left(p_{0}, q_{0}\right)$ to leak to the surrounding points.

Then, we consider the case of large number of rays, i.e., $\mathbf{G}_{k_{x}}=\iint_{\left(\theta_{k_{x}}, \varphi_{k_{x}}\right) \in \Delta_{k_{x}}} r_{x}\left(\theta_{k_{x}}, \varphi_{k_{x}}\right) \mathbf{A}\left(\theta_{k_{x}}, \varphi_{k_{x}}\right) d \theta_{k_{x}} d \varphi_{k_{x}}$. According to (13), it can be concluded that each ray of
$\mathbf{G}_{k_{x}}$ is corresponding to a (or limited) nonzero point as $N_{v}, N_{h} \rightarrow \infty$, and is restricted in a relatively small range under the narrow AS range condition, which is given by (14), shown at the top of the next page, where the $\mathcal{B}_{k_{x}}$ is called the beamspace of channel $\mathbf{G}_{k_{x}}$. This completes the proof of Lemma 2.

$$
\begin{gathered}
\mathcal{B}_{k_{x}}=\left\{(p, q)|| N_{v} \frac{d_{v}}{\lambda}\left(\cos \theta_{k_{x}}\right)_{\min }\right\rfloor \\
\leq p \leq\left\lceil N_{v} \frac{d_{v}}{\lambda}\left(\cos \theta_{k_{x}}\right)_{\max }\right\rceil,\left\lfloor N_{h} \frac{d_{h}}{\lambda}\left(\sin \theta_{k_{x}} \cos \varphi_{k_{x}}\right)_{\min }\right\rfloor \\
\leq q \leq\left\lceil N_{h} \frac{d_{h}}{\lambda}\left(\sin \theta_{k_{x}} \cos \varphi_{k_{x}}\right)_{\max }\right\rceil, \forall\left(\theta_{k_{x}}, \varphi_{k_{x}}\right) \\
\left.\in \Delta_{k_{x}}\right\}, \text { as } N_{v}, N_{h} \rightarrow \infty
\end{gathered}
$$

Based on Lemma 2, the channel matrix can be approximately represented by

$$
\mathbf{G}_{k_{x}}=\mathbf{F}_{N_{\nu}}^{H} \tilde{\mathbf{G}}_{k_{x}} \mathbf{F}_{N_{h}}^{H} \approx\left(\mathbf{F}_{N_{\nu}}^{H}\right)_{:, \Omega_{p}} \tilde{\mathbf{G}}_{k_{x}}^{\left\{\mathcal{B}_{k_{x}}\right\}}\left(\mathbf{F}_{N_{h}}^{H}\right)_{\Omega_{q},:}
$$

where $\Omega_{p}$ and $\Omega_{q}$ are the indexes of the columns and rows given by $\mathcal{B}_{k_{x}}$, and $\tilde{\mathbf{G}}_{k_{x}}^{\left\{\mathcal{B}_{k_{x}}\right\}}$ is the beamspace channel whose dimension has been efficiently reduced from $N_{v} \times N_{h}$ to $\left|\mathcal{B}_{k_{x}}\right|$, where $\left|\mathcal{B}_{k_{x}}\right|$ denotes the cardinality of the beamspace $\mathcal{B}_{k_{x}}$. Note that, according to Lemma 2 it can be obtained that (15) becomes equality when $N_{\nu}$ and $N_{h}$ tend to infinity. Then, the beamspace channel can be written as:

$$
\tilde{\mathbf{G}}_{k_{x}}^{\left\{\mathcal{B}_{k_{x}}\right\}}=\mathbf{F}_{N_{v}}^{\left\{\mathcal{B}_{k_{x}}^{\text {row }}\right\}} \mathbf{G}_{k_{x}} \mathbf{F}_{N_{h}}^{\left\{\mathcal{B}_{k_{x}}^{\text {col }}\right\}}
$$

where $\mathcal{B}_{k_{x}}^{\text {row }} \triangleq\left\{\left(\Omega_{p},:\right)\right\}$ and $\mathcal{B}_{k_{x}}^{\text {col }} \triangleq\left\{\left(:, \Omega_{q}\right)\right\}$ are called the vertical beamspace and horizontal beamspace which consist of the vertical beams and horizontal beams in $\mathcal{B}_{k_{x}}$, respectively.

Corollary 1 In the large $N_{v}$ and $N_{h}$ regime, if the beamspaces of user $k_{x}$ and $i_{x}(k \neq i, x \in\{u, d\})$ are nonoverlapping, then the BD channels of $k_{x}$ and $i_{x}$ are orthogonal, which mathematically can be expressed as

$\lim _{N_{v}, N_{h} \rightarrow \infty} \mathbf{F}_{N_{v}}^{\left\{\mathcal{B}_{i x}^{\text {row }}\right\}} \mathbf{G}_{k_{x}} \mathbf{F}_{N_{h}}^{\left\{\mathcal{B}_{i_{x}}^{\text {col }}\right\}}=\lim _{N_{v}, N_{h} \rightarrow \infty} \mathbf{F}_{N_{v}}^{\left\{\mathcal{B}_{k x}^{\text {row }}\right\}} \mathbf{G}_{i_{x}} \mathbf{F}_{N_{h}}^{\left\{\mathcal{B}_{k x}^{\text {col }}\right\}}=0$

The conclusion in Corollary 1 indicates that if the beamspaces of different users are orthogonal, the users in the $\mathrm{BD}$ are "invisible" to each other, i.e., the signals transmitted on their own beamspace channels won't affect other users. 
To illustrate the concept of beamspace, an example of DL BD channels $\tilde{\mathbf{G}}_{k_{d}}$ whose DoDs are $\left(21^{\circ}, 8^{\circ}\right),\left(41^{\circ}, 25^{\circ}\right)$, and $\left(70^{\circ}, 8^{\circ}\right)$ are depicted in Fig. 2. In most cases in the reality, the AS in the vertical domain is relatively small due to the height of the BS [32]; therefore, we set the AS in the horizontal directions $\delta_{h}$ is $10^{\circ}$ and in the vertical directions $\delta_{v}$ is $5^{\circ}$. Obviously, the channel power is concentrated on the beamspace, but with the finite number of antennas, the power might leak to the around points.
In order to facilitate the operation, we rewrite the BD channel into equivalent vectorial form as $\tilde{\mathbf{g}}_{k_{x}}=$ $\operatorname{vec}\left(\tilde{\mathbf{G}}_{k_{x}}\right)=\operatorname{vec}\left(\mathbf{F}_{N_{v}} \mathbf{G}_{k_{x}} \mathbf{F}_{N_{h}}\right)$. Using the property $\operatorname{vec}(\mathbf{A B C})=\left(\mathbf{C}^{T} \otimes \mathbf{A}\right) \operatorname{vec}(\mathbf{B})$, we can get

$$
\tilde{\mathbf{g}}_{k_{x}}=\left(\mathbf{F}_{N_{h}}^{T} \otimes \mathbf{F}_{N_{v}}\right) \mathbf{g}_{k_{x}}=\mathbf{F g}_{k_{x}}
$$

where $\mathbf{F}=\left(\mathbf{F}_{N_{h}}^{T} \otimes \mathbf{F}_{N_{v}}\right)$ and $x \in\{u, d\}$. Similarly, the beamspace channel vector is $\tilde{\mathbf{g}}_{k_{x}}^{\operatorname{vec}\left\{\mathcal{B}_{k_{x}}\right\}}=\operatorname{vec}\left(\tilde{\mathbf{G}}_{k_{x}}^{\left\{\mathcal{B}_{k_{x}}\right\}}\right)$,

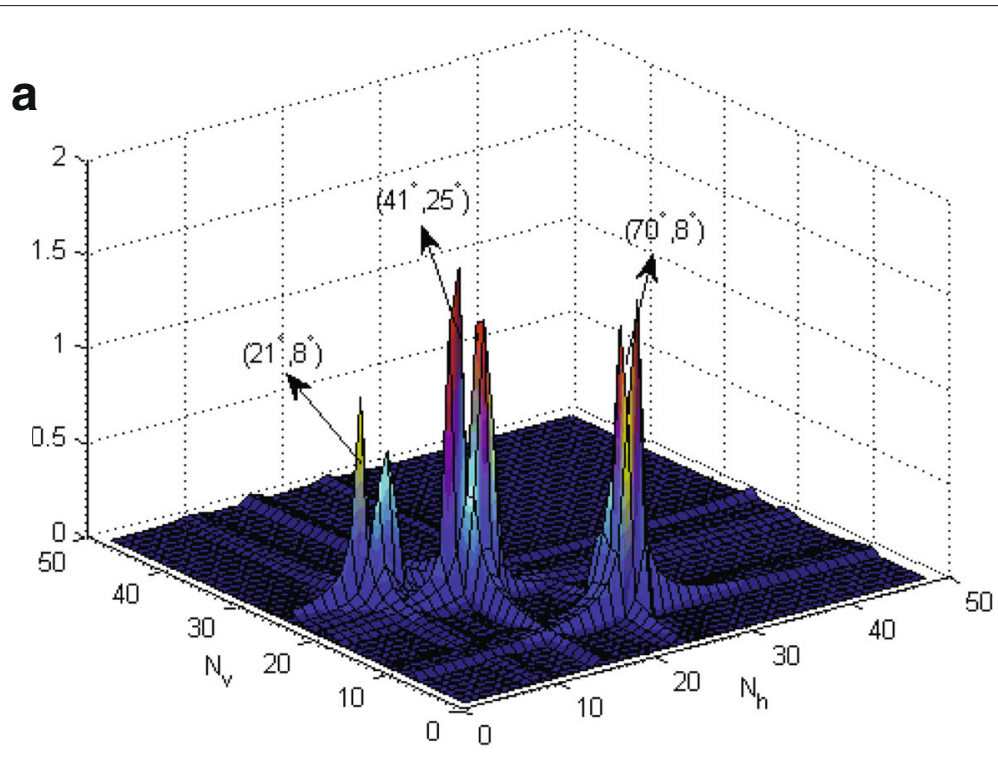

b

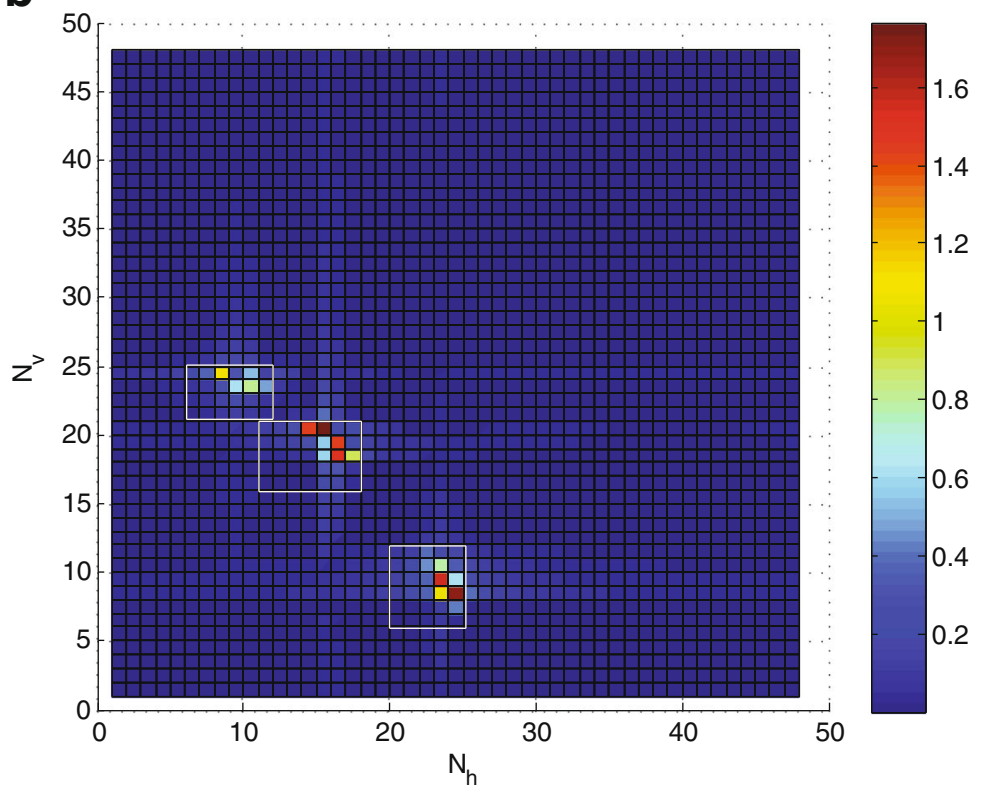

Fig. 2 Example of BD channels and their beamspaces with $N_{v}=N_{h}=48$, the AS in the horizontal directions $\delta_{h}$ is $10^{\circ}$ and in the vertical directions $\delta_{V}$ is $5^{\circ}$. a The BD channels $\tilde{\mathbf{G}}_{k_{d}}$ whose DoDs are $\left(21^{\circ}, 8^{\circ}\right),\left(41^{\circ}, 25^{\circ}\right)$, and $\left(70^{\circ}, 8^{\circ}\right)$. $\mathbf{b}$ The top view of $\mathbf{a}$ and the beamspace of each channel which is generated by (14) and is marked with rectangular frame 
where vec $\left\{\mathcal{B}_{k_{x}}\right\}$ is the vectorial beamspace which is denoted as $B_{k_{x}}$. Then, we have

$$
\tilde{\mathbf{g}}_{k_{x}}^{\left\{B_{k_{x}}\right\}}=\mathbf{F}^{\left\{B_{k_{x}}\right\}} \mathbf{g}_{k_{x}}=\left[\left(\mathbf{F}_{N_{h}}^{\left\{\mathcal{B}_{k_{x}}^{\text {col }}\right\}}\right)^{T} \otimes \mathbf{F}_{N_{v}}^{\left\{\mathcal{B}_{k_{x}}^{\text {row }}\right\}}\right] \mathbf{g}_{k_{x}} .
$$

From Lemma 2, we can see that, with the practical finite number of antennas, most power of the beamspace channel are concentrated around the points as (14) shows, and the dimension of beamspace is also related to the AS range of the signal rays. For keeping the required channel power of the beamspace channel, the dominant beams that are selected for the transmission at the user $k_{x}$ are defined as:

$$
\begin{aligned}
\mathcal{B}_{k_{x}}= & \left\{1 \leq i \leq N_{v}, 1 \leq j \leq N_{h}:\right. \\
& \left.\left|\left[\tilde{\mathbf{G}}_{k_{x}}\right]_{i, j}\right|^{2} \geq \xi_{k_{x}} \max _{i, j}\left|\left[\tilde{\mathbf{G}}_{k_{x}}\right]_{i, j}\right|^{2}\right\}
\end{aligned}
$$

where threshold $\xi_{k_{x}} \in(0,1)$ which can be selected so that $\tilde{\mathbf{G}}_{k_{x}}^{\left\{\mathcal{B}_{k_{x}}\right\}}$ captures a significant fraction $\rho$ of the power of $\tilde{\mathbf{G}}_{k_{x}}$ (e.g., $\rho \geq 0.9$ ). $\rho$ is the required effective power ratio close to 1 . Since $\mathcal{B}_{k_{x}}$ is only related to the direction information, i.e., $\left(\theta_{k_{x}}, \varphi_{k_{x}}\right)$, which varies over a relatively long time and can be accurately obtained at the BS with little hindrance through long-term feedback [33]. Therefore, we can preestablish an off-line data table of different $\left(\theta_{k_{x}}, \varphi_{k_{x}}\right)$ for the $\mathcal{B}_{k_{x}}$ in order to decrease the complexity of computation.

\subsection{Beamspace of SI channel}

In FD 3D massive MIMO systems, the dimension of SI channel is ultra-high $\left(N_{v} N_{h} \times N_{v} N_{h}\right)$; therefore, the compressibility of $\mathbf{G}_{S I}$ is urgent to be explored and exploited.

We first introduce an eigenvalue characteristic of largescale UPA. Using the eigenvalue decomposition, $\mathbf{C}_{a v}$ and $\mathbf{C}_{a h}$ can be expressed as:

$$
\begin{aligned}
& \mathbf{C}_{a v}=\mathbf{U}_{a v} \mathbf{D}_{a v} \mathbf{U}_{a v}^{H} \\
& \mathbf{C}_{a h}=\mathbf{U}_{a h} \mathbf{D}_{a h} \mathbf{U}_{a h}^{H}
\end{aligned}
$$

where $a \in\{R, T\}, \mathbf{U}_{a v}$ and $\mathbf{U}_{a h}$ are unitary matrices, $\mathbf{D}_{a v}=$ $\operatorname{diag}\left\{\lambda_{a v}^{(1)}, \cdots, \lambda_{a v}^{\left(N_{\nu}\right)}\right\}$ and $\mathbf{D}_{a h}=\operatorname{diag}\left\{\lambda_{a h}^{(1)}, \cdots, \lambda_{a h}^{\left(N_{h}\right)}\right\}$.

According to [12], for the large dimension of ULA, the eigenvector matrix can be approximated by a unitary DFT matrix. Since UPA is composed of ULAs in each row and column, and under the assumption of large dimension of antenna array at the BS, we can approximate the $\mathbf{C}_{a v}$ and $\mathbf{C}_{a h}$ as

$$
\begin{aligned}
& \mathbf{C}_{a v} \stackrel{N_{v} \rightarrow \infty}{=} \mathbf{F}_{N_{v}} \mathbf{D}_{a v} \mathbf{F}_{N_{v}}^{H}, \\
& \mathbf{C}_{a h} \stackrel{N_{h} \rightarrow \infty}{=} \mathbf{F}_{N_{h}} \mathbf{D}_{a h} \mathbf{F}_{N_{h}}^{H},
\end{aligned}
$$

and the eigenvalues $\mathbf{D}_{a v}$ and $\mathbf{D}_{a h}$ have the following characteristic:

$$
\begin{aligned}
& \mathbf{D}_{a v}=\operatorname{diag}\left\{0, \cdots, 0, \lambda_{a v}^{(i)}, \cdots, \lambda_{a v}^{(k)}, 0, \cdots, 0\right\} \\
& \mathbf{D}_{a h}=\operatorname{diag}\left\{0, \cdots, 0, \lambda_{a h}^{(t)}, \cdots, \lambda_{a h}^{(u)}, 0, \cdots, 0\right\}
\end{aligned}
$$

where $\lambda_{a v}$ and $\lambda_{a h}$ are non-negligible. Then, we can get the dominate domain of $\mathbf{D}_{a}$ as:

$$
\mathbf{D}_{a}^{\left\{\Lambda_{a}\right\}}=\operatorname{diag}\{\underbrace{\lambda_{a v}^{(i)} \lambda_{a h}^{(t)}, \cdots, \lambda_{a v}^{(i)} \lambda_{a h}^{(u)}, \cdots, \lambda_{a v}^{(k)} \lambda_{a h}^{(u)}}_{(k-i) \times(u-t)}\}
$$

where $\mathbf{D}_{a}=\mathbf{D}_{a h} \otimes \mathbf{D}_{a v}, \Lambda_{a}=\left\{\left(\Omega_{a}, \Omega_{a}\right) \mid m \in \Omega_{a}\right.$, $\left.\left[\mathbf{D}_{a}\right]_{m, m} \neq 0\right\}$ and $\Omega_{a}$ is the set that contains the indexes of rows (columns) of the dominate domain of $\mathbf{D}_{a}$.

In practical FD systems, the SI signal is the main bottleneck of further improving the SE; thus, minimizing the harmful effect of SI is the primary task. In order to realize 3D multiuser FD transmission, we first define the BD SI channel $\tilde{\mathbf{G}}_{S I}$ as $\tilde{\mathbf{G}}_{S I}=\mathbf{F}_{r} \mathbf{G}_{S I} \mathbf{F}_{t}^{H}$, where $\mathbf{F}_{r}$ and $\mathbf{F}_{t}$ can be equal to $\mathbf{F}$ or a submatrix of $\mathbf{F}$. Let $\Lambda_{a}^{\text {row }}=\left\{\left(\Omega_{a},:\right)\right\}$, $\Lambda_{a}^{\text {col }}=\left\{\left(:, \Omega_{a}\right)\right\}, \mathbf{P}=\mathbf{F}^{2}$ be a permutation matrix and $\mathbf{d}=\left(1,2, \cdots, N_{v} N_{h}\right)^{T}$ denotes the indexes of rows of $\mathbf{D}_{R}$. Then, the SI channel gain $\mathbb{E}\left[\left\|\mathbf{G}_{S I}\right\|^{2}\right]$ can be effectively weakened by the following lemma:

Lemma 3 Define $B_{T}=\Lambda_{T}^{\text {row }}$ and $B_{R}=$

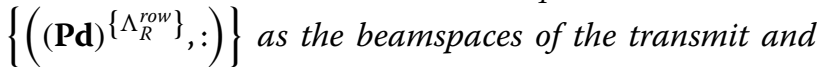
receive SI, then $\mathbb{E}\left[\left\|\tilde{\mathbf{G}}_{S I}\right\|^{2}\right]$ tends to zero if $\mathbf{F}_{t}=\mathbf{F}^{\left\{B_{u}\right\}}$ and $\mathbf{F}_{r}=\mathbf{F}^{\left\{B_{d}\right\}}$, where $B_{u} \cap B_{T}=\emptyset$ and $B_{d} \cap B_{R}=\emptyset$.

Proof See Appendix: Proof of Lemma 3.

\section{3D multiuser beamspace transmission scenario}

With 2D large-scale antenna arrays deployed at the BS, the spatial resolution of vertical and horizontal directions in 3D massive MIMO systems are high. We can exploit the slow time-varying angular information such as DoA, DoD, and channel correlation to efficiently estimate channels and transmit data. In this section, we detail our proposed 3D MUBT scheme. Based on user grouping scheme and beamspace channel estimation method, we show the scheme is capable of eliminating the SI, IGI, and MUI.

\subsection{Beamspace-aware user scheduling}

Due to the simultaneous transmission of UL and DL, the existence of SI and CCI restricts the overall performance of FD cellular systems. However, we can exploit the orthogonality of beamspace to avoid the SI, and by user scheduling strategy, the $\mathrm{CCI}$ can be ignored. To begin 
with, the user are partitioned into groups on the basis of the following criteria:

Criterion: Allocate the UL/DL users whose beamspaces are the same to the same group, while the beamspaces of different UL/DL groups are orthogonal, i.e.,

$$
B_{g_{i}^{u}} \cap B_{g_{j}^{u}}=\emptyset, B_{g_{i}^{d}} \cap B_{g_{j}^{d}}=\emptyset
$$

where $B_{g_{i}^{u}}$ and $B_{g_{j}^{u}}$ are the beamspaces of UL group $g_{i}^{u}$ and $g_{j}^{u}, B_{g_{i}^{d}}$ and $B_{g_{j}^{d}}$ are the beamspaces of DL group $g_{i}^{d}$ and $g_{j}^{d}$.

To simplify the illustration, the cardinalities of the beamspaces are set to be the same, i.e., $\left|B_{g_{i}^{u}}\right|=\left|B_{g_{j}^{u}}\right|=b_{u}$ and $\left|B_{g_{i}^{d}}\right|=\left|B_{g_{j}^{d}}\right|=b_{d}$. Assume that $K_{g_{i}^{x}}$ is the number of users in group $g_{i}^{x}$, and $g_{i, k}^{x}$ denotes the index of the $k$ th user in group $g_{i}^{x}$, where $x \in\{u, d\}$. In the FD cellular systems, if the co-channel UL and DL users are too close to each other, the CCI may cause a serious trouble to the DL users' signal-to-interference ratio. Therefore, we provide a simple user scheduling algorithm to mitigate the SI and attenuate the CCI. The main idea of our scheduling strategy to suppress the CCI is to locate the co-channel users apart. Within the cellular systems, the sufficient distance isolation between co-channel users can significantly reduce the $\mathrm{CCI}$ due to the plenty of obstructions such as buildings and trees, which as a result makes the CCI negligible compared to the signals transmitted from the high-altitude $\mathrm{BS}^{2}$
Firstly, we partition the cell into four areas $\mathcal{A}_{1}, \mathcal{A}_{2}, \mathcal{A}_{3}$, and $\mathcal{A}_{4}$ as Fig. 3 shows, then the user scheduling algorithm is proposed as the following.

Algorithm 1 User Scheduling Based on the Distance and SI Beamspaces Isolation

1: Calculate the beamspaces of all UL and DL users through (14) and (20).

2: Partition UL and DL users into groups according to the beamspace-aware grouping criterion (25). Let $\mathcal{G}_{u}$ and $\mathcal{G}_{d}$ be the set of UL and DL groups which satisfy $\mathcal{G}_{u}=\left\{g_{i}^{u} \mid B_{g_{i}^{u}} \cap B_{T}=\varnothing, i=1, \cdots, G_{u}\right\}$ and $\mathcal{G}_{d}=$ $\left\{g_{i}^{d} \mid B_{g_{i}^{d}} \cap B_{R}=\varnothing, i=1, \cdots, G_{d}\right\}$, respectively.

3: Schedule $G_{u}$ UL groups in location area $\mathcal{A}$. If $\mathcal{A}=$ $\mathcal{A}_{1} \mathcal{A}_{2}$, then schedule the DL users in $\mathcal{A}_{4}$; else if $\mathcal{A}=$ $\mathcal{A}_{3} \mathcal{A}_{4}$, then schedule the DL users in $\mathcal{A}_{1}$.

4: If all UL groups' beamspaces are orthogonal to $B_{T}$, then schedule $G_{d}$ DL groups; else, schedule $G_{d}$ DL groups from $\mathcal{G}_{d}$ so as to eliminate the SI in the beamspace of $g_{i}^{u}$.

5: To include all the possibility, in the orthogonal time/frequency resources, schedule $G_{d}$ DL groups, and use the similar method in step 3 to determine in which area the UL groups are scheduled. If all DL groups' beamspaces are orthogonal to $B_{R}$, then schedule $G_{u}$ UL groups; else, schedule $G_{u}$ UL groups from $\mathcal{G}_{u}$.

6: Repeat 3, 4, 5 .

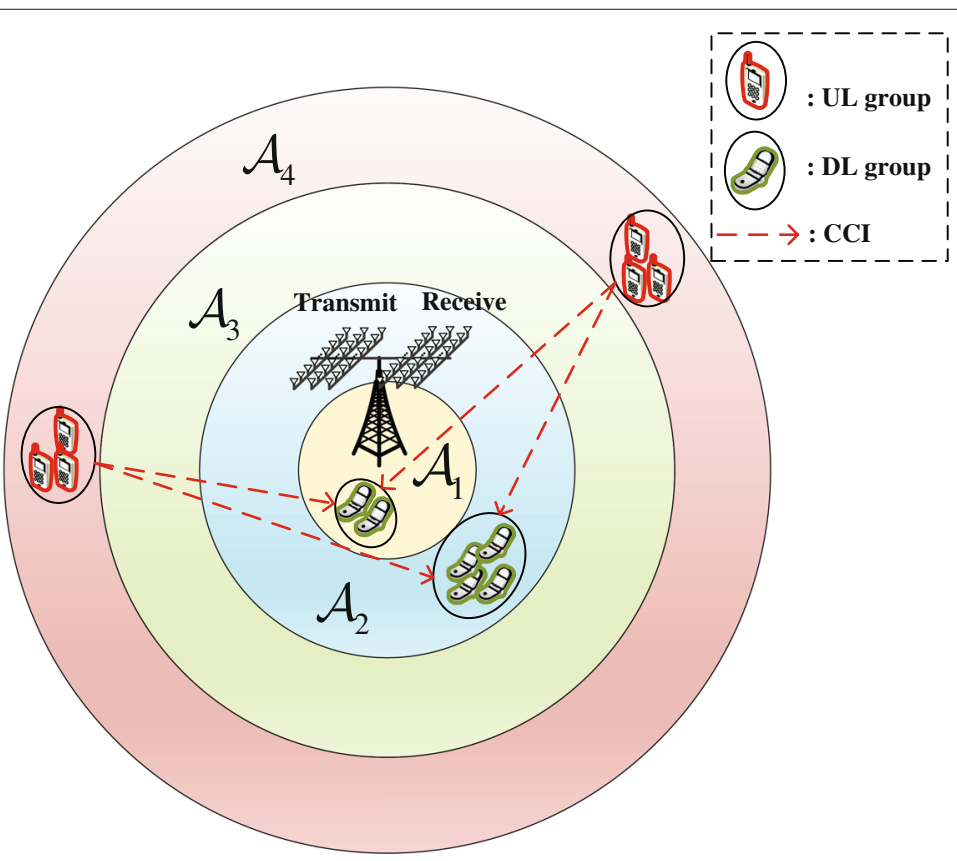

Fig. 3 Illustration of CCI between UL and DL groups under the cell partitioning scheme 


\subsection{Effective beamspace channel estimation}

During the estimation phase of the FD system, all UL users transmit the pilot sequences to the BS, and simultaneously, the BS transmits the pilot sequences to the DL users. Then, the received pilot signals at the $\mathrm{BS}$ and the $i$ th $\mathrm{DL}$ group are written as:

$$
\begin{gathered}
\mathbf{Y}_{u}=\sum_{i=1}^{G_{u}} \mathbf{G}_{g_{i}^{u}} \mathbf{X}_{g_{i}^{u}}+\mathbf{G}_{S I} \sum_{l=1}^{G_{d}} \tilde{\mathbf{X}}_{g_{l}^{d}}+\mathbf{N}_{u} \\
\mathbf{Y}_{g_{i}^{d}}=\mathbf{G}_{g_{i}^{d}}^{H} \sum_{i=1}^{G_{d}} \tilde{\mathbf{X}}_{g_{i}^{d}}+\sum_{j=1}^{G_{u}} \mathbf{H}_{g_{i}^{d}, g_{j}^{u}} \mathbf{X}_{g_{j}^{u}}+\mathbf{N}_{g_{i}^{d}}
\end{gathered}
$$

where $\mathbf{G}_{g_{i}^{u}}=\left[\mathbf{g}_{g_{i, 1}^{u}}, \cdots, \mathbf{g}_{g_{i, K_{g}}^{u}}\right] \in \mathbb{C}^{N_{v} N_{h} \times K_{g_{i}^{u}}}$ and $\mathbf{G}_{g_{i}^{d}}=$ $\left[\mathbf{g}_{g_{i, 1}^{d}}, \cdots, \mathbf{g}_{g_{i, K}^{d}}\right] \in \mathbb{C}^{N_{v} N_{h} \times K_{g_{i}^{d}}}$ are the channel matrices between the BS and the $i$ th UL and DL user group, respectively. $\mathbf{H}_{g_{i}^{d}, g_{j}^{u}}$ is the co-channel matrix between $j$ th $\mathrm{UL}$ group and $i$ th DL group. The second term of the right side of (27) is the CCI resulting from the simultaneous $\mathrm{UL}$ and DL transmission at the same frequency. Based on the user scheduling algorithm, the CCI can be ignored in the FD scenario, which as a result is omitted in the later analysis. $\mathbf{X}_{g_{i}^{u}} \in \mathbb{C}^{K_{g_{i}^{u}} \times \tau_{u}}$ is pilot sequences of the $i$ th UL group, $\tau_{u}$ denotes the length of pilot sequence for each UL user, $\tilde{\mathbf{X}}_{g_{i}^{d}}=\left({ }_{\mathbf{F}}\left\{{ }^{B} g_{i}^{d}\right\}\right){ }^{H} \mathbf{X}_{g_{i}^{d}}$ denotes the precoded DL pilot sequence where $\mathbf{X}_{g_{i}^{d}} \in \mathbb{C}^{b_{d} \times \tau_{d}}$ is the DL beamspace pilot sequences for the $i$ th DL group, $\tau_{d}$ is the length of DL pilot sequence, $\mathbf{N}_{u}$ and $\mathbf{N}_{g_{i}^{d}}$ are the additive white Gaussian noise (AWGN) matrices which have independent and identically distributed $\mathcal{C} N(0,1)$ elements. In order to reduce the overhead of channel estimation, we can reuse the same pilot sequences between different groups. Let $\mathbf{X}_{u} \in \mathbb{C}^{1 \leq i \leq G_{u}} K_{g_{i}^{u}} \times \tau_{u}$ and $\mathbf{X}_{d} \in \mathbb{C}^{b_{d} \times \tau_{d}}$ be the orthogonal UL pilot sequence set and DL pilot sequence set which satisfy $\mathbf{X}_{a} \mathbf{X}_{a}^{H}=\tau_{a} P_{p}^{a} \mathbf{I}, a \in\{u, d\}$, where $P_{p}^{a}$ is the transmit power of each pilot symbol and $\mathbf{I}$ is the identity matrix with appropriate size. Then, the UL and DL pilot sequences assigned to the different groups are assumed to share the same UL and DL pilot sequence set. Mathematically, the UL pilot sequences allocated for group $g_{i}^{u}$ is given by $\mathbf{X}_{g_{i}^{u}}=\mathbf{X}_{u}^{\left\{1: K_{g_{i}^{u}}, ;\right\}}$, and the DL pilot sequences allocated for group $g_{i}^{d}$ is given by $\mathbf{X}_{g_{i}^{d}}=\mathbf{X}_{d}^{\left\{: 1: \tau_{u}\right\}}$ if $\tau_{d} \geq \tau_{u}$ and $\mathbf{X}_{g_{i}^{d}}=\left[\mathbf{X}_{d} \mathbf{0}_{b_{d} \times\left(\tau_{u}-\tau_{d}\right)}\right]$ if $\tau_{d} \leq \tau_{u}$.

\subsubsection{UL beamspace channel estimation}

In order to eliminate the IGI, which is also the pilot contamination, we multiply both sides of (26) with $\mathbf{F}\left\{B_{g_{i}^{u}}\right\}$ and we have:

$$
\begin{aligned}
\tilde{\mathbf{Y}}_{g_{i}^{u}}=\mathbf{G}_{g_{i}^{u}}^{\left\{B_{g_{i}^{u}}\right\}} \mathbf{X}_{g_{i}^{u}}+\sum_{j=1, j \neq i}^{G_{u}} \mathbf{G}_{g_{j}^{u}}^{\left\{B_{g_{i}^{u}}\right\}} \mathbf{X}_{g_{j}^{u}}+\mathbf{F}^{\left\{B_{g_{i}}\right\}} \mathbf{N}_{u} \\
+\underbrace{\left.\sum_{l=1}^{G_{d}} \mathbf{F}^{\left\{B_{g_{i}^{u}}\right\}} \mathbf{G}_{S I}\left(\left.\mathbf{F}\right|^{\left.B_{g_{l}}\right\}}\right\}\right)^{H} \mathbf{X}_{g_{l}^{d}}}_{\text {SI }} .
\end{aligned}
$$

Then, the BS obtains the observation of UL beamspace channel as:

$$
\begin{aligned}
\mathbf{y}_{g_{i, k}^{u}}^{\mathrm{ob}}= & \tilde{\mathbf{g}}_{g_{i, k}^{u}}^{\left\{B_{g_{i}^{u}}\right\}}+\sum_{j=1, j \neq i}^{G_{u}} \tilde{\mathbf{g}}_{g_{j, k}^{u}}^{\left\{B_{g_{i}^{u}}\right\}}+\frac{1}{\tau_{u} P_{p}^{u}}\left(\mathbf{F}^{\left\{B_{g_{i}^{u}}^{u}\right\}}\right)^{H} \mathbf{N}_{u} \mathbf{X}_{g_{i}^{u}}^{H} \mathbf{e}_{k} \\
& +\underbrace{\frac{1}{\tau_{u} P_{p}^{u}} \sum_{l=1}^{G_{d}} \mathbf{F}\left\{{ }^{B_{g_{i}^{u}}^{u}} \mathbf{G}_{S I}\left(\mathbf{F}\left\{{ }^{B} g_{l}^{d}\right\}\right)\right)^{H} \tilde{\mathbf{X}}_{g_{l}} \mathbf{X}_{g_{i}^{u}}^{H} \mathbf{e}_{k}}_{\text {SI }}
\end{aligned}
$$

The second term of the right side of (29) is the pilot contamination between the $k$ th users in all UL groups due to the reuse of the same pilot sequence. Note that, according to the grouping criterion and Corollary 1, the pilot contamination approaches to zero as $N_{v}, N_{h} \rightarrow \infty$. The last term is the SI caused by the simultaneous UL and DL training. Note that if users are scheduled by Algorithm 1, according to Lemma 3, the SI can be wiped out in the large $N_{v}, N_{h}$ regime. Based on the observation in (29), the minimum mean square error (MMSE) estimation of the beamspace channel $\tilde{\mathbf{g}}_{g_{i, k}^{u}}^{\left\{B_{g_{i}^{u}}\right\}}$ can be obtained as:

$$
\hat{\tilde{\mathbf{g}}}_{g_{i, k}^{u}}^{\left\{B_{g_{i}^{u}}\right\}}=\mathbf{R}_{g_{i, k}^{u}}^{\left\{B_{g_{i}^{u}}\right\}} \mathbf{C}_{k_{u}}^{-1} \mathbf{y}_{g_{i, k}^{u}}^{\mathrm{ob}}
$$

where $\mathbf{R}_{g_{i, k}^{u}}^{\left\{B_{g_{i}}\right\}}$ is given by:

$$
\begin{aligned}
& \mathbf{R}_{g_{i, k}^{u}}^{\left\{B_{g_{i}}^{u}\right\}}=\iint \mathbf{F}^{\left\{B_{g_{i}^{u}}^{u}\right\}}\left(\mathbf{b}\left(h_{g_{i, k}^{u}}\right) \mathbf{b}^{H}\left(h_{g_{i, k}^{u}}^{u}\right)\right) \\
& \otimes\left(\mathbf{a}\left(v_{g_{i, k}^{u}}\right) \mathbf{a}_{g_{i, k}^{u}}\right) \in \Delta_{k_{u}} \\
& \left.\times S_{u}\left(v_{g_{i, k}^{u}}\right)\right)\left(\mathbf{F}^{\left\{B_{g_{i}}^{u}\right\}}, \varphi_{g_{i, k}^{u}}\right) d \theta_{g_{i, k}^{u}} d \varphi_{g_{i, k}^{u}}^{H}
\end{aligned}
$$


where $S_{u}\left(\theta_{g_{i, k}^{u}}^{u}, \varphi_{i, k}^{u}\right)=r_{u}\left(\theta_{g_{i, k}^{u}}, \varphi_{g_{i, k}^{u}}\right) r_{u}^{H}\left(\theta_{g_{i, k}^{u}}, \varphi_{g_{i, k}^{u}}\right)$, and

$$
\begin{aligned}
\mathbf{C}_{k_{u}}= & \sum_{l=1}^{G_{u}} \mathbf{R}_{g_{l, k}^{u}}^{\left\{B_{g_{i}^{u}}\right\}}+\frac{1}{\tau_{u} P_{p}^{u}} \mathbf{I} \\
& +\frac{1}{\left(\tau_{u} P_{p}^{u}\right)^{2}} \sum_{j=1}^{G_{d}} \sum_{l=1}^{G_{d}} \mathbf{F}^{\left.B_{g_{i}^{u}}\right\}^{\prime}} \mathbf{G}_{S I}\left(\mathbf{F}\left\{{ }^{B} g_{l}^{d}\right\}\right)^{H} \tilde{\mathbf{X}}_{g_{l}^{d}} \mathbf{X}_{g_{i}^{u}}^{H} \mathbf{e}_{k} \\
& \times \mathbf{e}_{k}^{H} \mathbf{X}_{g_{i}^{u}} \tilde{\mathbf{X}}_{g_{j}^{d}}^{H} \mathbf{F}^{\left\{B_{g_{j}^{d}}\right\}} \mathbf{G}_{S I}^{H}\left(\mathbf{F}^{\left\{g_{g_{i}^{u}}\right\}}\right)^{H}
\end{aligned}
$$

Based on the orthogonal property of MMSE estimation [34], the channel eatimation error is $\overline{\tilde{\mathbf{g}}}_{g_{i, k}^{u}}^{\left\{B_{g_{i}}\right\}}=\tilde{\mathbf{g}}_{g_{i, k}^{u}}^{\left\{g_{i}^{u}\right\}}$ $\hat{\tilde{\mathbf{g}}}{ }_{g_{i, k}^{u}}^{\left\{B_{g_{i}^{u}}\right\}}$, and the covariance of $\overline{\tilde{\mathbf{g}}}_{g_{i, k}^{u}}^{\left\{B_{g_{i}}\right\}}$ is:

$$
\overline{\mathbf{R}} g_{i, k}^{u}=\mathbf{R}_{g_{i, k}^{u}}^{\left\{B_{g_{i}^{u}}^{u}\right\}}-\mathbf{R}_{g_{i, k}^{u}}^{\left\{B_{g_{i}^{u}}^{u}\right\}} \mathbf{C}_{k_{u}}^{-1} \mathbf{R}_{g_{i, k}^{u}}^{\left\{B_{g_{i}^{u}}^{u}\right\}} .
$$

\subsubsection{DL beamspace channel estimation}

(27) can be rewritten as:

$$
\mathbf{Y}_{g_{i}^{d}}=\left(\mathbf{G}_{g_{i}^{d}}^{\left\{{ }_{g_{i}^{d}}\right\}}\right)^{H} \mathbf{X}_{g_{i}^{d}}+\underbrace{\sum_{j=1, j \neq i}^{G_{d}}\left(\mathbf{G}_{g_{i}^{d}}\left\{{ }_{g_{j}^{d}}\right\}\right.}_{\text {pilot contamination }})^{H} \mathbf{X}_{g_{j}^{d}}+\mathbf{N}_{g_{i}^{d}} .
$$

Then, the observation of the DL beamspace channel is written as

$$
\begin{aligned}
& \mathbf{y}_{g_{i, k}^{d}}^{\mathrm{ob}}=\frac{1}{\tau_{d} P_{p}^{d}}\left(\mathbf{Y}_{g_{i}^{d}} \mathbf{X}_{g_{i}^{d}}^{H}\right)^{H} \mathbf{e}_{k} \\
& =\tilde{\mathbf{g}}_{g_{i, k}^{d}}^{\left\{B_{g_{i}^{d}}\right\}}+\underbrace{\sum_{j=1, j \neq i}^{\sum_{d}} \tilde{\mathbf{g}}_{g_{i, k}^{d}}\left\{B_{g_{j}^{d}}\right\}}_{\text {pilot contamination }}+\frac{1}{\tau_{d} P_{p}^{d}}\left(\mathbf{N}_{g_{i}^{d}} \mathbf{X}_{g_{i}^{d}}^{H}\right)^{H} \mathbf{e}_{k}
\end{aligned}
$$

The second term of the right side of (35) is the pilot contamination caused by the reuse of the same DL pilot sequence set over all DL groups, and it tends to vanish as $N_{v}, N_{h} \rightarrow \infty$ according to the grouping criterion and Corollary 1 .

Using the similar method as UL estimation, we have:

$$
\hat{\tilde{\mathbf{g}}}_{g_{i, \mathrm{k}}^{d}}^{\left\{B_{g_{i}^{d}}\right\}}=\mathbf{R}_{g_{i, k}^{d}}^{\left\{B_{g_{i}^{d}}\right\}} \mathbf{C}_{k_{d}}^{-1} \mathbf{y}_{g_{i, k}^{d}}^{\mathrm{ob}}
$$

where

$$
\begin{aligned}
& \mathbf{R}_{g_{i, k}^{d}}^{\left\{B_{g_{i}^{d}}\right\}}=\sum_{l=1}^{G_{d}} \iint_{\left(\theta_{g_{i, k}^{d}}, \varphi_{g_{i, k}^{d}}^{d}\right) \in \Delta_{k_{d}}} \mathbf{F}\left\{{ }^{\left.B_{g_{i}^{d}}\right\}}\left(\mathbf{b}\left(h_{g_{i, k}^{d}}\right) \mathbf{b}^{H}\left(h_{g_{i, k}^{d}}\right)\right)\right. \\
& \otimes\left(\mathbf{a}\left(v_{g_{i, k}^{d}}\right) \mathbf{a}^{H}\left(v_{g_{i, k}^{d}}\right)\right)\left(\mathbf{F}\left\{B_{g_{l}^{d}}\right\}\right)^{H} \\
& \times S_{d}\left(\theta_{g_{i, k}^{d}}, \varphi_{g_{i, k}^{d}}\right) d \theta_{g_{i, k}^{d}} d \varphi_{g_{i, k}^{d}}
\end{aligned}
$$

and

$$
\begin{aligned}
& \mathbf{C}_{k_{d}}=\sum_{j=1}^{G_{d}} \mathbf{R}_{g_{i, k}^{d}}^{\left\{B_{g_{j}^{d}}\right\}}+\frac{1}{\tau_{d} P_{p}^{d}} \mathbf{I} \\
& =\sum_{j=1}^{G_{d}} \sum_{l=1}^{G_{d}} \iint{ }_{\left({ }_{g_{i, k}^{d}}^{d}, \varphi_{g_{i, k}^{d}}\right) \in \Delta_{k_{d}}} \mathbf{F}^{\left.B_{g_{j}^{d}}\right\}}\left(\mathbf{b}\left(h_{g_{i, k}^{d}}\right) \mathbf{b}^{H}\left(h_{g_{i, k}^{d}}\right)\right) \\
& \otimes\left(\mathbf{a}\left(v_{g_{i, k}^{d}}\right) \mathbf{a}^{H}\left(v_{g_{i, k}^{d}}\right)\right)\left(\mathbf{F}\left\{{ }^{B} g_{l}^{d}\right\}\right)^{H} \\
& \times S_{d}\left(\theta_{g_{i, k}^{d}}, \varphi_{g_{i, k}^{d}}\right) d \theta_{g_{i, k}^{d}} d \varphi_{g_{i, k}^{d}}+\frac{1}{\tau_{d} P_{p}^{d}} \mathbf{I} .
\end{aligned}
$$

Similarly, the covariance of DL channel estimation error $\overline{\tilde{\mathbf{g}}}_{g_{i, k}^{d}}\left\{\begin{array}{l}\left.B_{g_{i}^{d}}\right\} \\ \text { is }\end{array}\right.$

$$
\overline{\mathbf{R}}_{g_{i, k}^{d}}^{\left\{B_{g_{i}^{d}}\right\}}=\mathbf{R}_{g_{i, k}^{d}}^{\left\{B_{g_{i}}\right\}}-\mathbf{R}_{g_{i, k}^{d}}^{\left\{B_{g_{i}^{d}}\right\}} \mathbf{C}_{k_{d}}^{-1} \mathbf{R}_{g_{i, k}^{d}}^{\left\{B_{g_{i}^{d}}\right\}} .
$$

\subsection{Data transmission}

At the data transmission stage ${ }^{3}$, UL and DL users transmit signals in the same time-frequency resource. In our transmission scheme, the data are transmitted in each group's own beamspace. In the BD, the received signal at the BS from the $i$ th UL group and the received signal at the $i$ th DL group can be written as:

$$
\begin{aligned}
\tilde{\mathbf{y}}_{g_{i}^{u}}=\tilde{\mathbf{G}}_{g_{i}^{u}}^{\left\{B_{g_{i}^{u}}\right\}} \mathbf{s}_{g_{i}^{u}}+\underbrace{\sum_{j=1, j \neq i}^{G_{u}} \tilde{\mathbf{G}}_{g_{j}^{u}}^{\left\{B_{g_{i}^{u}}\right\}} \mathbf{s}_{g_{j}^{u}}+\tilde{\mathbf{n}}_{g_{i}^{u}}}_{\mathrm{IGI}} \\
+\underbrace{\sum_{j=1}^{G_{d}} \mathbf{F}^{\left\{B_{g_{i}^{u}}\right\}} \mathbf{G}_{S I}\left(\mathbf{F}^{\left\{B_{g_{j}}\right\}}\right)^{H} \mathbf{x}_{g_{i}^{d}}}_{\mathrm{SI}} .
\end{aligned}
$$




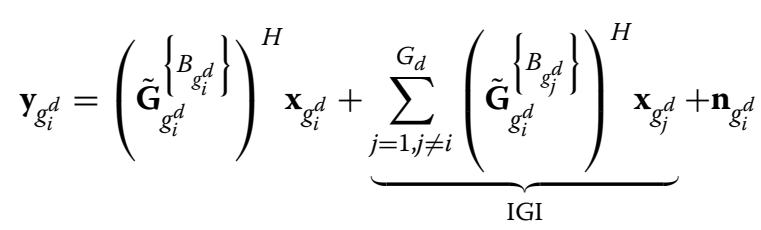

where $\mathbf{s}_{g_{i}^{u}} \in \mathbb{C}^{K_{g_{i}^{u}} \times 1}$ and $\mathbf{x}_{g_{i}^{d}} \in \mathbb{C}^{b_{d} \times 1}$ denote the transmit data of the $i$ th UL group and the BD precoded data for users in the $i$ th DL group, respectively, and $\tilde{\mathbf{n}}_{g_{i}^{u}}=$ $\mathbf{F}\left\{B_{g_{i}^{u}}\right\} \mathbf{n}_{g_{i}^{u}}$. Note that the IGIs in (40) and (41) tend to zero as $N_{v}, N_{h} \rightarrow \infty$.

For massive MIMO systems, linear signal processing techniques have already been shown to have good performance [2]. Therefore, in this paper, define $\mathbf{W}_{g_{i}^{u}}=\left[\mathbf{w}_{g_{i, 1}^{u}}, \cdots, \mathbf{w}_{g_{i, K_{g}^{u}}^{u}}\right] \in \mathbb{C}^{b_{u} \times K_{g_{i}^{u}}}$ and $\mathbf{w}_{g_{i}^{d}}=$ $\left[\mathbf{w}_{g_{i, 1}^{d}}, \cdots, \mathbf{w}_{g_{i, K}^{d} g_{g_{i}^{d}}}\right] \in \mathbb{C}^{b_{d} \times K_{g_{i}^{d}}}$ as the linear UL detector and DL precoder at the BS. Multiplying (40) with $\mathbf{W}_{g_{i}^{u}}^{H}$ we have $\mathbf{y}_{g_{i}^{u}}=\mathbf{W}_{g_{i}^{u}}^{H} \tilde{\mathbf{y}}_{g_{i}^{u}}$. Then, the received signal of user $g_{i, k}^{u}$ in beamspace $B_{g_{i}^{u}}$ can be written as

$$
\begin{aligned}
& y_{g_{i, k}^{u}}^{u}=\mathbf{w}_{g_{i, k}^{u}}^{H} \tilde{\mathbf{g}}_{g_{i, k}^{u}}^{\left\{B_{g_{i}^{u}}\right\}} s_{g_{i, k}^{u}}+\underbrace{\mathbf{w}_{g_{i, k}^{u}}^{H} \sum_{k^{\prime}=1, k^{\prime} \neq k}^{K_{g_{i}^{u}}^{u}} \tilde{\mathbf{g}}_{g_{i, k^{\prime}}}^{\{}\left\{s_{g_{i}^{u}}\right\} s_{g_{i, k^{\prime}}^{u}}}_{\text {MUI }}
\end{aligned}
$$

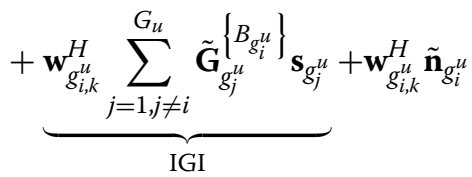

$$
\begin{aligned}
& +\underbrace{\mathbf{w}_{g_{i, k}^{u}}^{H} \sum_{l=1}^{K_{g_{d}}} \mathbf{F}^{\left\{B_{g_{i}^{u}}\right\}} \mathbf{G}_{S I}\left(\left.\mathbf{F}\right|^{\left.B_{g_{l}^{d}}\right\}}\right)^{H} \mathbf{W}_{g_{l}^{d}} \mathbf{s}_{g_{l}^{d}}}_{\text {SI }}
\end{aligned}
$$

which is capable of decoding the data of user $g_{i, k}^{u}$, and the second term of the right side of (42) is the MUI within the group. For DL, the BD precoded data can be written as $\mathbf{x}_{g_{i}^{d}}=\mathbf{W}_{g_{i}^{d}} \mathbf{s}_{g_{i}^{d}}$; substituting it into (41), we can express the received signal at the user $g_{i, k}^{d}$ in $B_{g_{i}^{d}}$ as:

$$
\begin{aligned}
y_{g_{i, k}^{d}}= & \left(\tilde{\mathbf{g}}_{g_{i, k}^{d}}^{\left\{B_{g_{i}^{d}}\right\}}\right)^{H} \mathbf{w}_{g_{i, k}^{d}} S_{g_{i, k}^{d}}+\underbrace{\left.\sum_{j=1, j \neq i}^{G_{d}}\left(\tilde{\mathbf{g}}_{g_{i, k}^{d}} B_{g_{j}^{d}}\right\}\right) \mathbf{w}_{g_{j}^{d}} \mathbf{s}_{g_{j}^{d}}}_{\text {IGI }} \\
& +\underbrace{\left.\left(\tilde{\mathbf{g}}_{g_{i, k}^{d}} B_{g_{i}^{d}}\right\}\right)^{H} \sum_{k^{\prime}=1, k^{\prime} \neq k}^{K_{g_{i}^{d}}} \mathbf{w}_{g_{i, k^{d}}} S_{g_{i, k^{\prime}}}+n_{g_{i, k}}}_{\mathrm{MUI}}
\end{aligned}
$$

We adopt the zero-forcing (ZF) beamforming scheme to eliminate the MUI. The CSI of UL and DL beamspace channels is assumed to be obtained by (30) and (36); then, the beamforming matrices can be expressed as:

$$
\begin{aligned}
& \mathbf{W}_{g_{i}^{u}}=\hat{\tilde{\mathbf{H}}}_{g_{i}^{u}}^{\left\{B_{g_{i}^{u}}\right\}}\left(\left(\hat{\tilde{\mathbf{H}}}_{g_{i}^{u}}^{\left\{B_{g_{i}^{u}}\right\}}\right)^{H} \hat{\tilde{\mathbf{H}}}_{g_{i}^{u}}^{\left.B_{g_{i}^{u}}\right\}}\right)^{-1} \\
& \mathbf{W}_{g_{i}^{d}}=\hat{\tilde{\mathbf{H}}}_{g_{i}^{d}}^{\left\{B_{g_{i}^{d}}\right\}}\left(\left(\hat{\tilde{\mathbf{H}}}_{g_{i}^{d}}^{\left\{B_{g_{i}}\right\}}\right)^{H} \hat{\tilde{\mathbf{H}}}_{g_{i}^{d}}^{\left\{B_{g_{i}^{d}}\right\}}\right)^{-1} \Gamma_{g_{i}^{d}}-\frac{1}{2}
\end{aligned}
$$

where $\Gamma_{g_{i}^{d}}=\operatorname{diag}\left\{\beta_{i, 1}, \beta_{i, 2}, \cdots, \beta_{i, K_{g_{i}^{d}}}\right\} \in \mathbb{C}^{K_{g_{i}^{d}} \times K_{g_{i}^{d}}}$ is a diagonal matrix for the normalization of the precoder.

Based on (42) and according to [35], we can obtain the signal-to-interference-plus-noise ratio (SINR) of user $g_{i, k}^{u}$ as (45), shown at the top of the next page, where $P_{g_{i, k}^{u}}^{u}$ is the transmit power of user $g_{i, k}^{u}, \mathbf{P}_{g_{j}^{u}}=\mathbb{E}\left[\mathbf{s}_{g_{j}^{u}} \mathbf{s}_{g_{j}^{u}}^{H}\right]$ and $\mathbf{P}_{g_{l}^{d}}=\mathbb{E}\left[\mathbf{s}_{g_{l}} \mathbf{s}_{g_{l}^{d}}^{H}\right]$ are the transmit power matrices, $\Psi_{l}=$ $\left.\mathbf{F}^{\left\{B_{g_{i}}^{u}\right\}} \mathbf{G}_{S I}\left(\mathbf{F}^{\left\{B_{g_{l}}\right\}}\right)\right)^{H} \mathbf{W}_{g_{l}^{d}}$. According to (43) and the similar method in [35], the SINR of user $g_{i, k}^{d}$ can be expressed as (46), shown at the top of next page.

$$
\gamma_{g_{i, k}^{u}}^{u}=\frac{P_{g_{i, k}^{u}}}{\mathbf{w}_{g_{i, k}^{u}}^{H}\left[\sum_{k^{\prime}=1}^{K_{g_{i}^{u}}^{u}} P_{g_{i, k^{\prime}}^{u}} \Delta \tilde{\mathbf{g}}_{g_{i, k^{\prime}}^{u}}^{\left\{B_{g_{i}^{u}}^{u}\right\}}\left(\Delta \tilde{\mathbf{g}}_{g_{i, k^{\prime}}^{u}}^{\left\{B_{g_{i}^{u}}^{u}\right\}}\right)^{H}+\sum_{j=1, j \neq i}^{G_{u}} \tilde{\mathbf{G}}_{g_{j}^{u}}\left\{B_{g_{i}^{u}}^{u}\right\} \mathbf{P}_{g_{j}^{u}}\left(\tilde{\mathbf{G}}_{g_{j}^{u}}^{\left\{B_{g_{i}^{u}}\right\}}\right)^{H}+\sum_{l=1}^{G_{d}} \Psi_{l} \mathbf{P}_{g_{l}^{d}} \Psi_{l}^{H}+\mathbf{I}_{b_{u}}\right] \mathbf{w}_{g_{i, k}^{u}}}
$$




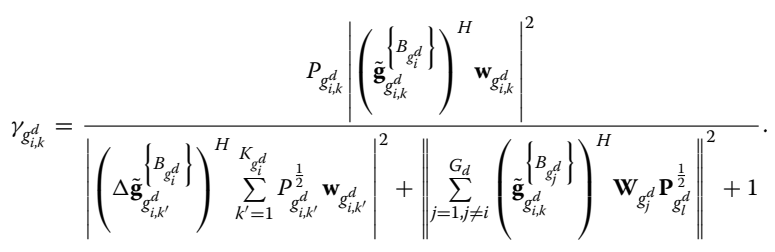

Eventually, the achievable rates of user $g_{i, k}^{u}$ and $g_{i, k}^{d}$ are expressed as:

$$
R_{g_{i, k}^{a}}=\frac{T-\max \left(\tau_{u}, \tau_{d}\right)}{T} \mathbb{E}\left[\log _{2}\left(1+\gamma_{g_{i, k}^{a}}\right)\right], a \in\{u, d\}
$$

\section{Simulation results}

In this section, we present the performance of the proposed scheme through simulation results. The parameters of the simulated single-cell multi-user systems are listed in Table 1. For path loss, we consider the 3GPP LTE simulation model [36]. It is assumed that the transmit and receive antenna arrays are separated in distance and some passive SIC techniques, such as radio frequency absorber material, are adopted so that the level of residual SI $\beta_{S I}=15 \mathrm{~dB}$. We consider that both the UL and DL users are scheduled by Algorithm 1 and are gathered into three groups. We assume that each group has 8 users and the range of DoA or DoD of users within each group is the same.

\subsection{Channel estimation}

In order to evaluate the performance of our proposed channel estimation method, we define the average individual MSE as the performance metric which is given by:

$$
\kappa_{a}=\frac{1}{K_{a}} \sum_{i=1}^{G_{a}} \sum_{k=1}^{K_{g_{i}^{a}}} \operatorname{tr}\left(\overline{\mathbf{R}}_{g_{i, k}^{a}}^{\left\{B_{g_{i}}\right\}}\right), a \in\{u, d\}
$$

Figures 4 and 5 present the comparison of the UL and DL

\begin{tabular}{|c|c|}
\hline \multicolumn{2}{|l|}{ Parameters values } \\
\hline Path loss between BS and users & $\begin{array}{l}2.7+42.8 \log _{10}(L)[\mathrm{dB}], \mathrm{L} \text { : distance } \\
\text { in meter }\end{array}$ \\
\hline $\begin{array}{l}\text { Number of UL and DL users } \\
K_{d}=K_{u}\end{array}$ & 24 \\
\hline Antenna array size $N_{h} \times N_{V}$ & $25 \times 25$ \\
\hline $\begin{array}{l}\text { UPA array element spacing } \\
d_{h}=d_{v}\end{array}$ & $\lambda / 2$ \\
\hline $\begin{array}{l}\text { AS in the horizontal directions } \\
\delta_{h}\end{array}$ & $10^{\circ}$ \\
\hline AS in the vertical directions $\delta_{v}$ & $5^{\circ}$ \\
\hline $\begin{array}{l}\text { Required effective channel } \\
\text { power ratio } \rho\end{array}$ & 0.9 \\
\hline Channel coherence time $T$ & 200 symbols \\
\hline
\end{tabular}
channel estimation performance between the proposed

Table 1 Simulation parameters beamspace channel estimation and the conventional fulldimension channel estimation as a function of average UL and DL receive SNR, respectively. In our proposed HD/FD beamspace channel estimation scheme, the length of pilot sequence is related to the dimension of beamspace; hence, we set $\tau_{u}=\tau_{d}=\tau=\max \left\{b_{u}, b_{d}\right\}$. Under this setup, the default length of pilot sequence is $\tau=42$. We also compare the cases of $\tau=54$ and $\tau=72$. From the numerical results, we can see that the DL performance of the proposed scheme outperforms the conventional full-dimension method and the UL performance of the proposed scheme is better in the low-SNR region. What is more, comparing the proposed HD and FD scenarios, we can observe that in the low-SNR case, the performance is very close between $\mathrm{HD}$ and FD. As the SNR grows, the harmful impact of SI increases, which brings down the performance of FD scenario.

Note that, in the HD massive MIMO systems, the required minimum length of pilot sequence in the conventional TDD systems with DL reciprocity [5] and FDD systems [12] are $\sum_{i=1}^{G_{u}} K_{g_{i}^{u}}+\sum_{i=1}^{G_{d}} K_{g_{i}^{d}}=48$ and $\sum_{i=1}^{G_{u}} K_{g_{i}^{u}}+$ $b_{d}=66$ (approximate), respectively. In the FD massive MIMO systems, [24] assumes that transmit and receive radio-frequency chains share the transmit antennas at the BS so that DL CSI can be obtained by utilizing the reciprocity which is at the cost of hardware, and the required minimum length of pilot sequence is $\sum_{i=1}^{G_{u}} K_{g_{i}}+$ $\sum_{i=1}^{G_{d}} K_{g_{i}^{d}}=48$. In addition, we know that the computational complexity of MMSE estimation is $\mathcal{O}\left(n^{3}\right)$ due to the matrix inversion operation where $n$ is the dimension of the channel covariance matrix, which lays a heavy burden on the system if $n$ is large, especially for massive MIMO system. For example, the computational complexity of the conventional full-dimension MMSE estimation [6] is $\mathcal{O}\left(\left(N_{v} N_{h}\right)^{3}\right)=\mathcal{O}\left(625^{3}\right)$, which is very high. Nevertheless, the complexity of our low-dimension beamspace channel estimation scheme is $\mathcal{O}\left(\left[\max \left(b_{u}, b_{d}\right)\right]^{3}\right)$ which is $\mathcal{O}\left(30^{3}\right)$. From this perspective, our 3D MUBT scheme is competitive in 3D massive MIMO system application.

\subsection{Data transmission}

The SE in this subsection is defined as $S E=S E_{u}+S E_{d}$ where $S E_{a}=\frac{1}{2} \sum_{i=1}^{G_{a}} \sum_{k=1}^{K_{g_{i}}} \frac{T-\tau_{a}}{T} \log _{2}\left(1+\gamma_{g_{i, k}^{a}}\right), a \in\{u, d\}$, the factor $\frac{1}{2}$ is for the HD mode which does not exist in the FD case. To validate the feasibility and illustrate the effectiveness of the FD 3D MUBT scheme, Fig. 6 compares the SEs versus average receive SNR in different scenarios. It can be seen that the FD 3D MUBT achieves the best performance both in the low SNR and high SNR region. The reason is that even though assuming the DL reciprocity, the training overhead in [24] is still larger than the proposed 


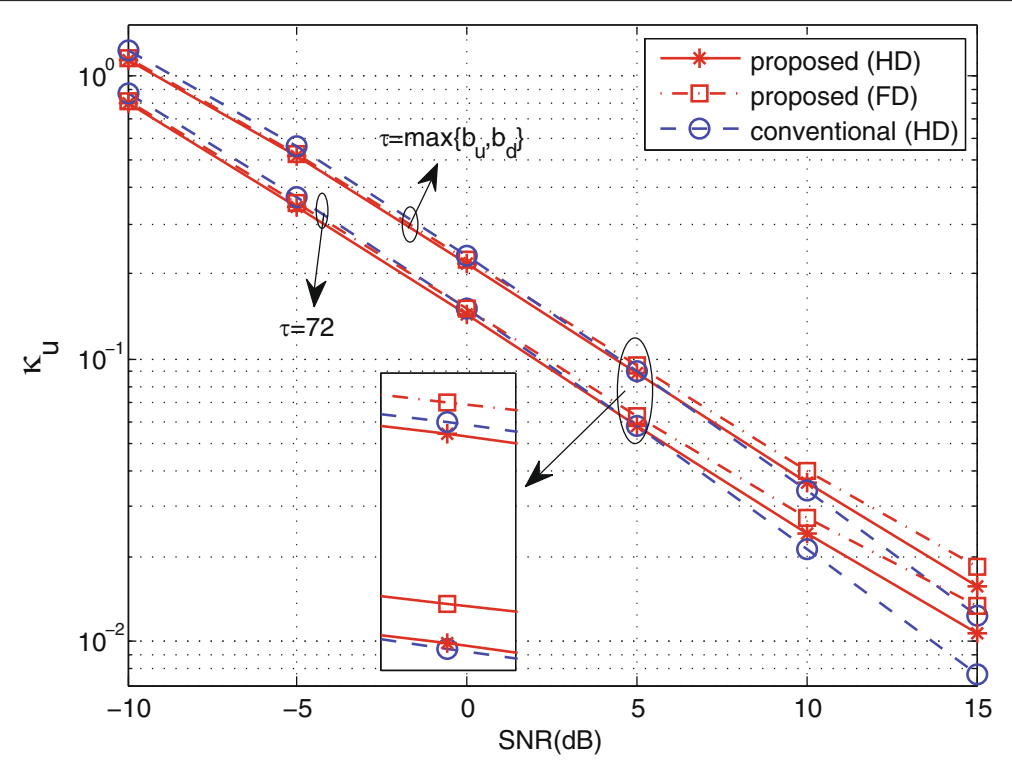

Fig. 4 Comparison of the UL channel estimation performance between the beamspace channel estimation and conventional estimation versus average receive SNRS

scheme. Besides, by using the user scheduling algorithm, the anti-SI capability of the proposed scheme outperforms the linear transceiver. Nevertheless, as the SNR grows, the performance gap between the FD 3D MUBT scheme and the FD scheme with linear transceiver decreases, and that between the HD 3D MUBT scheme and TDD case increases. The reason of them both is that as the SNR increases, the truncation error limits the performance of the proposed 3D MUBT scheme. What is more, the HD 3D MUBT outperforms the FDD [12] mainly due to the less training overhead.

Meantime, the comparisons that with less number of antennas $(15 \times 15)$ and no user scheduling are made. As expected, with less number of antennas, the overlap of beamspaces will increase which will increase the IGI and SI. Similarly, without the user scheduling algorithm, the

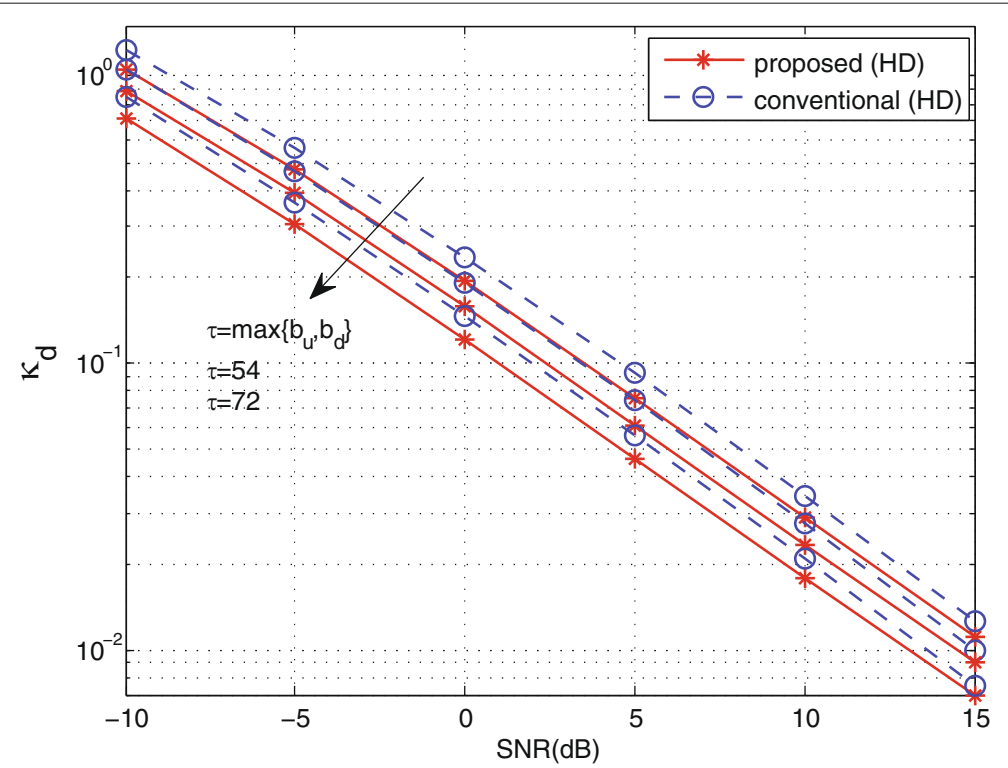

Fig. 5 Comparison of the DL channel estimation performance between the beamspace channel estimation and conventional estimation versus average receive SNRS 


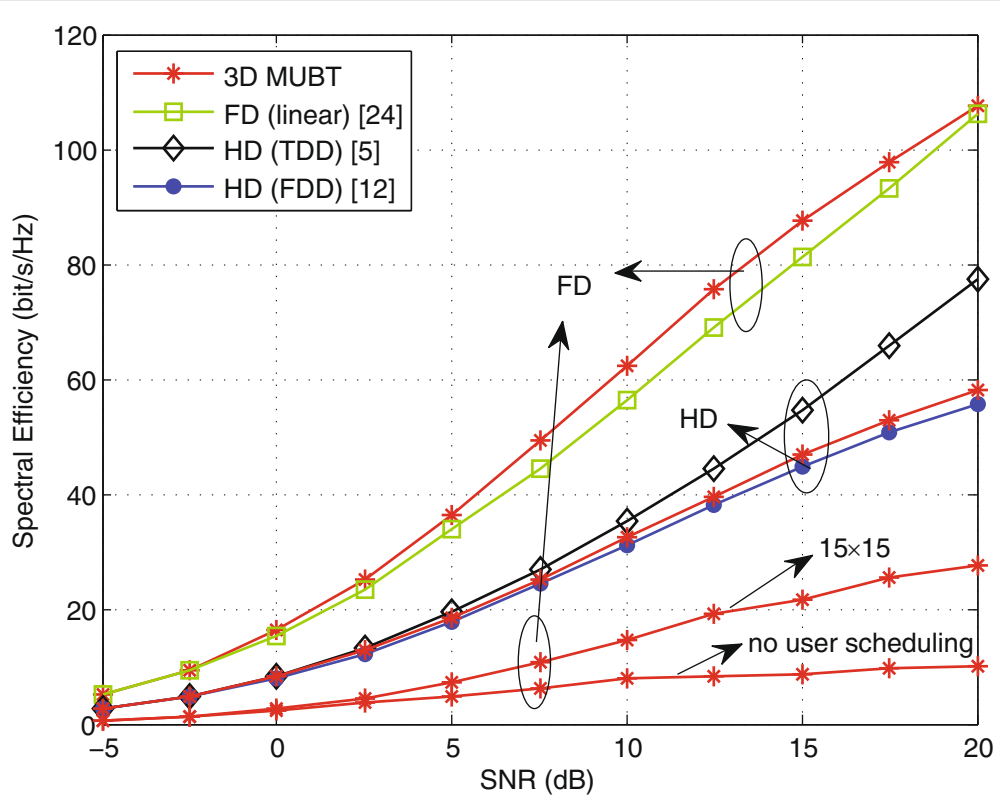

Fig. 6 SEs versus average receive SNRs in different scenarios

orthogonality of beamspaces between different groups of simultaneously scheduled users and SI cannot be guaranteed. And the CCI will also damage the system.

In the previous simulations, the $\beta_{S I}$ is set to the fixed value. To investigate how the SI level influences the SE performance in the FD massive MIMO systems, we compare the performance between HD system and FD system with different SI level $\beta_{S I}$ in Fig. 7. As expected, the performance of FD systems outperform that of HD systems when $\beta_{S I}$ level is low and reduces as $\beta_{S I}$ grows. It is observed that in the low $\beta_{S I}$ regions, the linear FD scheme achieves the better SE performance, which is because when the SI is relatively small, the channel estimation error, IGI and AWGN restrict the SE performance mainly; then, as a result, the linear scheme outperforms the proposed scheme due to the truncation error. For

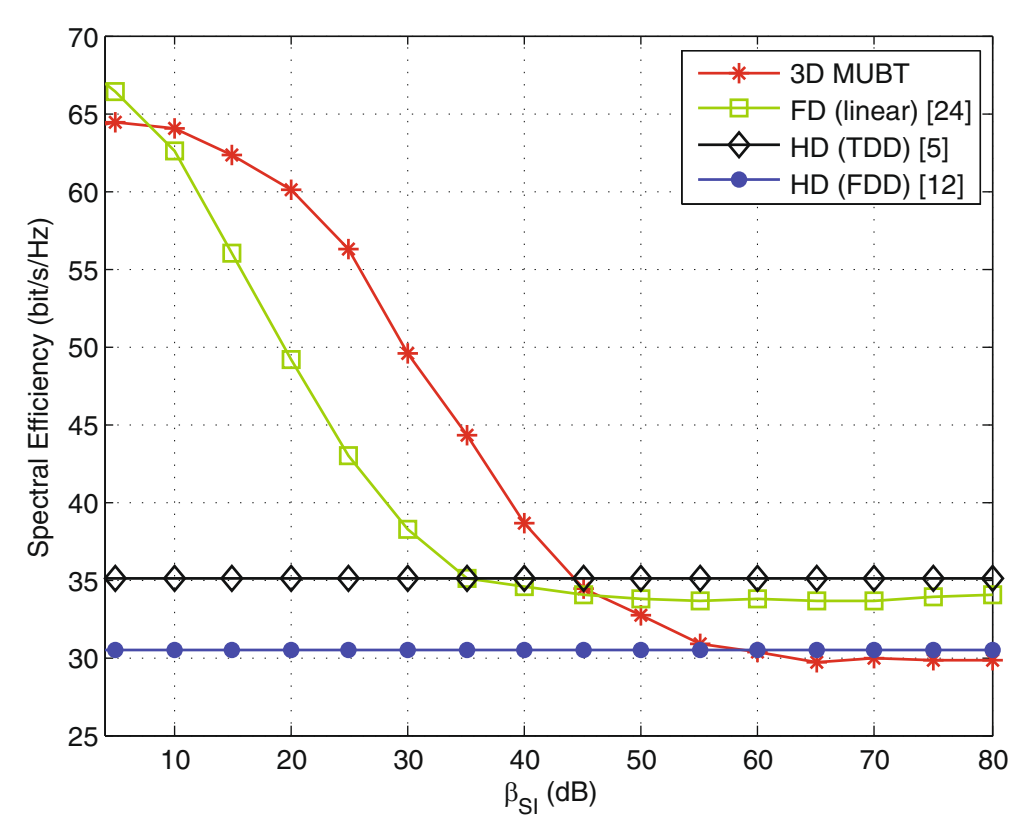

Fig. 7 SEs versus $\beta_{S I}$ in different scenarios with average receive $S N R=10 \mathrm{~dB}$ 


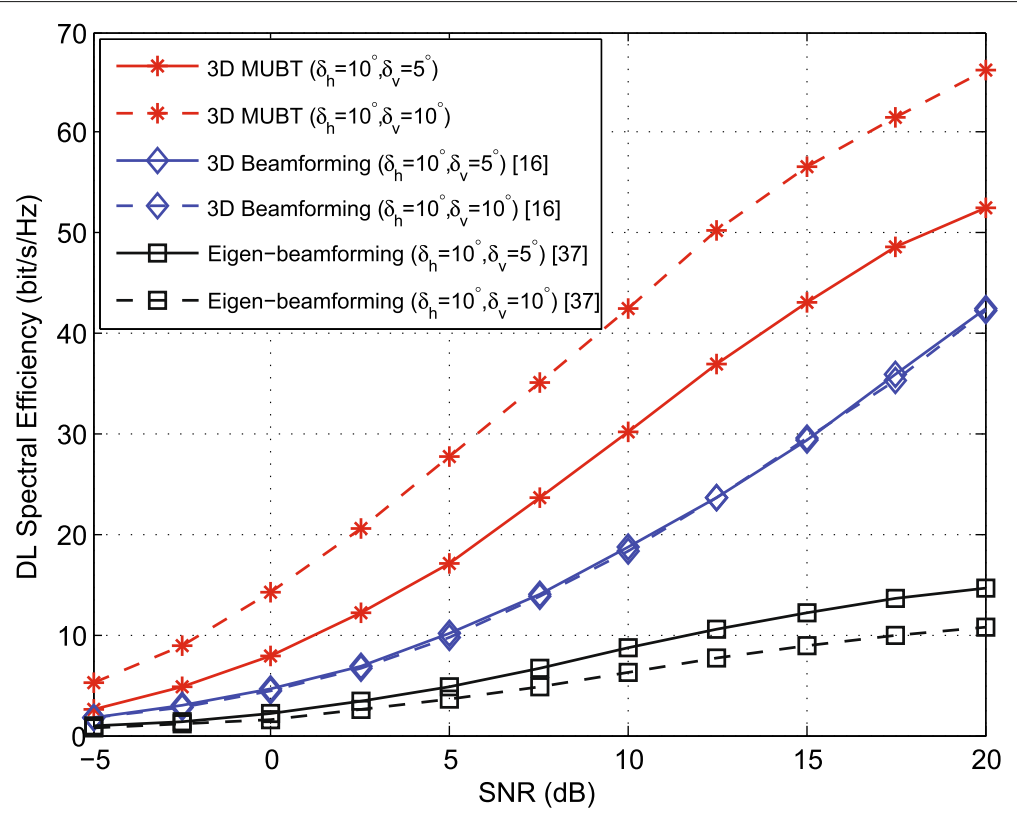

Fig. 8 SEs versus average DL receive SNRs in different 3D precoding scenarios

the similar reason, when the SI becomes the dominate, the impact of the SI on the two schemes is almost the same; therefore, the linear FD is superior when the SI level is high. However, when the $\beta_{S I}$ is within $(8,45)$, the FD 3D MUBT scheme outperforms the linear FD, which indicates that with the user scheduling strategy, the FD 3D MUBT scheme has better anti-SI capability. In other words, applying the FD 3D MUBT scheme can lower the requirement for the SIC techniques. Take $S E=49$ $\mathrm{bit} / \mathrm{s} / \mathrm{Hz}$ for example, the corresponding $\beta_{S I}$ for the FD scheme with linear transceiver and the FD 3D MUBT scheme are 20 and $30 \mathrm{~dB}$, which indicates that the SIC capability in conventional FD system needs $10 \mathrm{~dB}$ more than that in the proposed scheme and will bring the extra hardware cost and complexity.

\subsection{D precoding schemes}

According to [14], the channel correlation matrix can be well approximated by the Kronecker production of the correlations in horizontal and vertical directions, i.e., $\mathbf{C}=$ $\mathbf{C}_{h} \otimes \mathbf{C}_{v}$. Based on this, [37] designs a eigen-beamforming scheme. This scheme first chooses the principle eigenvector of $\mathbf{C}_{v}$, to perform the vertical dimension beamforming, then use the eigenvector matrix of $\mathbf{C}_{h}$ to do the horizontal dimension beamforming. In [16], Li et al. exploit the eigenvalue characteristic of large-scale UPA as discussed in Section 5.2 (23), and select the optimal DL beamforming vector for the $k$ th user as $\mathbf{b}_{k}=\left(\mathbf{F}_{N_{h}}\right)_{:, h} \otimes\left(\mathbf{F}_{N_{v}}\right)_{:, m}$ to maximize the average signal-to-leakage-plus-noise ratio, where $n$ and $m$ are the indexes of columns where the maximum eigenvalues of $\mathbf{C}_{h}$ and $\mathbf{C}_{v}$ are, respectively. In Fig. 8, we investigate the DL transmission with different $3 \mathrm{D}$ precoding schemes under the different conditions of $\delta_{v}$. Here, we get rid of the $1 / 2$ pre-log factor of the DL SE for the sake of illustration. We can see that the proposed scheme achieves the best performance. The performance of eigenbeamforming scheme decreases when $\delta_{v}$ increase. This is because the power of the channel is concentrated around the dominant eigenmode, and as the vertical AS increase, the power of the principle eigenvalue decrease. Moreover, the 3D beamforming scheme is insensitive to $\delta_{v}$ under this setup ${ }^{4}$. However, we can see that the SE performance of the proposed scheme increase as $\delta_{v}$ grows; this can be explained by (14). The dimension of the beamspace channel increases as AS grows while keep the distance between the beamspaces of groups and the IGI at acceptable levels, which as a result improves the SE.

\section{Conclusions}

In this paper, we propose a 3D MUBT scheme for FD cellular systems. By adopting the property of beamspace, the 3D MUBT scheme can not only efficiently mitigate the SI due to FD transmission, but also significantly reduce the overhead of channel estimation. The simulation results show that the proposed 3D MUBT scheme outperforms the FD scheme with linear transceiver and the HD (TDD/FDD) schemes in massive MIMO cellular system.

\section{Endnotes}

${ }^{1}$ Note that in FD techniques, the antenna configuration can also be the shared type, which only needs one 
antenna array to accomplish transmission and reception [19]. Nevertheless, due to the serious cross-talk within the antennas, it is impractical in MIMO system for now.

${ }^{2}$ We assume that the radius of the cell is $1000 \mathrm{~m}$. According to the 3GPP LTE BS-to-user and user-to-user path loss models for macrocell environment [36], the path loss between BS to users and users to users are PL $=2.7+$ $42.8 \log _{10}\left(R_{\mathrm{BS}}-\mathrm{UE}\right)$ and $\mathrm{PL}=55.78+40 \log _{10}\left(R_{\mathrm{UE}}-\mathrm{UE}\right)$, respectively, where $R_{\mathrm{BS} \text { - UE }}$ and $R_{\mathrm{UE} \text { - UE }}$ are distances in meter. When the distance between BS and users is $400 \mathrm{~m}$ (the shortest distance between users and users is $350 \mathrm{~m}$ according to the Algorithm 1), the interference channel between the two users is about $43 \mathrm{~dB}$ weaker than the useful channel.

${ }^{3}$ The estimated DL CSI feedback process is also important and is studied in a lot of literatures $[11,38,39]$. Choi et al. [39] shows that the influence of channel feedback noise and errors can be made negligible with respect to the channel estimation errors especially when the SNR is high, hence in this paper we consider the ideal DL CSI feedback for simplicity, as assumed in [12].

${ }^{4}$ In fact, the simulation results in [16] have the similar conclusion with the eigen-beamforming scheme, but when the number of user in each group is small, the decrease of the SE performance is inconspicuous.

\section{Appendix: Proof of Lemma 3}

Since both $\mathbf{F}_{N_{v}}$ and $\mathbf{F}_{N_{h}}$ are unitary, then $\mathbf{F}$ is a unitary matrix, based on which we can obtain

$$
\mathbb{E}\left[\left\|\tilde{\mathbf{G}}_{S I}\right\|^{2}\right]=\mathbb{E}\left[\left\|\mathbf{F}_{r} \mathbf{G}_{S I} \mathbf{F}_{t}^{H}\right\|^{2}\right]=\mathbb{E}\left[\left\|\mathbf{G}_{S I}\right\|^{2}\right]
$$

The vectorized BD SI channel is $\mathbf{g}_{S I}=\operatorname{vec}\left(\mathbf{G}_{S I}\right)$, and the vectorized BD SI channel is $\tilde{\mathbf{g}}_{S I}=\operatorname{vec}\left(\tilde{\mathbf{G}}_{S I}\right)$. According to (7), (8), and (22), $\mathbf{g}_{S I}$ can be represented as

$$
\begin{aligned}
\mathbf{g}_{S I} & =\operatorname{vec}\left(\mathbf{G}_{S I}\right)=\left(\mathbf{C}_{T}^{\frac{1}{2}} \otimes \mathbf{C}_{R}^{\frac{1}{2}}\right) \operatorname{vec}\left(\mathbf{G}_{w}\right) \\
& =\left(\mathbf{C}_{T h}^{\frac{1}{2}} \otimes \mathbf{C}_{T v}^{\frac{1}{2}}\right) \otimes\left(\mathbf{C}_{R h}^{\frac{1}{2}} \otimes \mathbf{C}_{R v}^{\frac{1}{2}}\right) \mathbf{g}_{w} \\
& \approx\left(\mathbf{F}_{N_{h}} \mathbf{D}_{T h}^{\frac{1}{2}} \mathbf{F}_{N_{h}}^{H}\right) \otimes\left(\mathbf{F}_{N_{v}} \mathbf{D}_{T v}^{\frac{1}{2}} \mathbf{F}_{N_{v}}^{H}\right) \otimes\left(\mathbf{F}_{N_{h}} \mathbf{D}_{R h}^{\frac{1}{2}} \mathbf{F}_{N_{h}}^{H}\right) \\
& \otimes\left(\mathbf{F}_{N_{v}} \mathbf{D}_{R v}^{\frac{1}{2}} \mathbf{F}_{N_{v}}^{H}\right) \mathbf{g}_{w} \\
& =\left(\mathbf{F D}_{T}^{\frac{1}{2}} \mathbf{F}^{H}\right) \otimes\left(\mathbf{F D}_{R}^{\frac{1}{2}} \mathbf{F}^{H}\right) \mathbf{g}_{w}
\end{aligned}
$$

Based on (49) and the property $\mathbb{E}\left[\left\|\tilde{\mathbf{G}}_{S I}\right\|^{2}\right]=$ $\mathbb{E}\left[\left\|\tilde{\mathbf{g}}_{S I}\right\|^{2}\right]$, we can get

$$
\begin{aligned}
& \mathbb{E}\left[\left\|\tilde{\mathbf{G}}_{S I}\right\|^{2}\right]=\mathbb{E}\left[\left\|\operatorname{vec}\left(\mathbf{F}_{r} \mathbf{G}_{S I} \mathbf{F}_{t}^{H}\right)\right\|^{2}\right]=\mathbb{E}\left[\left\|\left(\mathbf{F}_{t}^{*} \otimes \mathbf{F}_{r}\right) \mathbf{g}_{S I}\right\|^{2}\right] \\
& \stackrel{N_{v}, N_{h} \rightarrow \infty}{=} \mathbb{E}\left[\left\|\left(\mathbf{F}_{t}^{*} \otimes \mathbf{F}_{r}\right)\left(\mathbf{F D}_{T}^{\frac{1}{2}} \mathbf{F}^{H}\right) \otimes\left(\mathbf{F D}_{R}^{\frac{1}{2}} \mathbf{F}^{H}\right) \mathbf{g}_{w}\right\|^{2}\right]
\end{aligned}
$$

Then, we have

$$
\begin{aligned}
& \mathbb{E}\left[\left\|\tilde{\mathbf{G}}_{S I}\right\|^{2}\right] \stackrel{N_{v}, N_{h} \rightarrow \infty}{=} \mathbf{g}_{w}^{H}\left(\mathbf{F D}_{T}^{\frac{1}{2}} \mathbf{F}^{H} \mathbf{F}_{t}^{T}\right) \otimes\left(\mathbf{F D}_{R}^{\frac{1}{2}} \mathbf{F}^{H} \mathbf{F}_{r}^{H}\right) \\
& \times\left(\mathbf{F}_{t}^{*} \mathbf{F D}_{T}^{\frac{1}{2}} \mathbf{F}^{H}\right) \otimes\left(\mathbf{F}_{r} \mathbf{F D}_{R}^{\frac{1}{2}} \mathbf{F}^{H}\right) \mathbf{g}_{w}
\end{aligned}
$$

According to (29), $\mathbb{E}\left[\left\|\tilde{\mathbf{G}}_{S I}\right\|^{2}\right]$ can be effectively represented. Take the last two brackets of the right side of (34) for example, which is denoted as $I_{1}$ and $I_{2}$, we have:

$$
I_{1}=\mathbf{F}_{t}^{*} \mathbf{F D}_{T}^{\frac{1}{2}} \mathbf{F}^{H} \approx\left(\mathbf{F}^{\left\{B_{u}\right\}}\right)^{*} \mathbf{F}^{\left\{\Lambda_{T}^{\text {col }}\right\}}\left(\mathbf{D}_{T}^{\frac{1}{2}}\right)^{\left\{\Lambda_{T}\right\}}\left(\mathbf{F}^{H}\right)^{\left\{\Lambda_{T}^{\text {row }}\right\}}
$$

and

$$
I_{2}=\mathbf{F}_{r} \mathbf{F D}_{R}^{\frac{1}{2}} \mathbf{F}^{H} \approx \mathbf{F}^{\left\{B_{d}\right\}} \mathbf{F}^{\left\{\Lambda_{R}^{\text {col }}\right\}}\left(\mathbf{D}_{R}^{\frac{1}{2}}\right)^{\left\{\Lambda_{R}\right\}}\left(\mathbf{F}^{H}\right)^{\left\{\Lambda_{R}^{\text {row }}\right\}}
$$

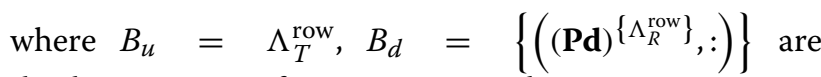
the beamspaces of transmit SI and receive SI, respectively, $\mathbf{P}=\mathbf{F}^{2}$ is the permutation matrix and $\mathbf{d}=$ $\left(1,2, \cdots, N_{v} N_{h}\right)^{T}$ denotes the indexes of rows of $\mathbf{D}_{R}$. It can be observed that (53) and (54) become equality as $N_{\nu}$ and $N_{h}$ tend to infinity. The other brackets can be similarly approximated as $I_{1}$ and $I_{2}$ do. In that way, if $B_{u} \cap B_{T}=\emptyset$ and $B_{d} \cap B_{R}=\emptyset$ are satisfied, $\mathbb{E}\left[\left\|\tilde{\mathbf{G}}_{S I}\right\|^{2}\right]$ will approach zero as $N_{v}, N_{h} \rightarrow \infty$. The proof is completed.

\section{Abbreviations}

2D: Two-dimensional; 3D: Three-dimensional; AS: Angular spread; BS: Base station; CCl: Co-channel interference; CSI: Channel state information; DoA: Direction of arrival; DoD: Direction of departure; DL: Downlink; FD: Full-duplex; FDD: Frequency-division duplex; HD: Half-duplex; ICl: Inter-cell interference; |Gl: Inter-group interference; LOS: Light-of-sight; MIMO: Multiple-input multipleoutput; MMSE: Minimum mean square error; MUBT: Multiuser beamspace transmission; MUI: Multiuser interference; NLOS: Non-ligth-of-sight; SE: Spectral efficiency; SI: Self-interference; SIC: SI cancellation; SINR: Signal-to-interferenceplus-noise ratio; TDD: Time-division duplex; UL: Uplink; ULA: Uniform linear antenna array; UPA: Uniform planar antenna array; ZF: Zero-forcing

\section{Funding}

This research was supported by the National Natural Science Foundation of China (No. 61671472), Jiangsu Province Natural Science Foundation 
(BK20160079), and National Natural Science Foundation of China (No. 61371123, No. 91438115).

\section{Authors' contributions}

$\mathrm{XW}$ is the main writer of this paper. He proposed the main idea, conducted the simulations, and analyzed it. DZ, KX, and WM assisted the review of this manuscript. All authors read and approved the final manuscript.

\section{Competing interests}

The authors declare that they have no competing interests.

\section{Publisher's Note}

Springer Nature remains neutral with regard to jurisdictional claims in published maps and institutional affiliations.

\section{Author details}

${ }^{1}$ Army Engineering University of PLA, No. 88, Houbiaoying Street, Nanjing, China. ${ }^{2}$ Unit 32020 of PLA, Wuhan, China.

Received: 20 April 2018 Accepted: 30 July 2018

Published online: 16 August 2018

\section{References}

1. J Mietzner, R Schober, L Lampe, WH Gerstacker, PA Hoeher, Multipleantenna techniques for wireless communications - a comprehensive literature survey. IEEE Commun. Surv. Tutorials. 11(2), 87-105 (2009)

2. TMarzetta, Noncooperative cellular wireless with unlimited numbers of base station antennas. IEEE Trans. Wirel. Commun. 9(11), 3590-3600 (2010)

3. HQ Ngo, E Larsson, T Marzetta, Energy and spectral efficiency of very large multiuser MIMO systems. IEEE Trans. Commun. 61(4), 1436-1449 (2013)

4. F Fernandes, A Ashikhmin, TL Marzetta, Inter-cell interference in noncooperative TDD large scale antenna systems. IEEE J. Sel. Areas Commun. 31(2), 192-201 (2013)

5. J Hoydis, S Brink, M Debbah, Massive MIMO in UL/DL of cellular networks: how many antennas do we need. IEEE J. Sel. Areas Commun. 31(2), 160-171 (2013)

6. L You, X Gao, XG Xia, N Ma, Y Peng, Pilot reuse for massive MIMO transmission over spatially correlated rayleigh fading channels. IEEE Trans. Wireless Commun. 14(6), 3352-3366 (2015)

7. H Cui, L Song, B Jiao, Multi-pair two-way amplify-and-forward relaying with very large number of relay antennas. IEEE Trans. Wireless Commun. 13(5), 2636-2645 (2014)

8. RCD Lamare, Massive MIMO systems: signal processing challenges and future trends. URSI Radio Science Bulletin. 86(4), 8-20 (2017)

9. J Jose, A Ashikhmin, TL Marzetta, S Vishwanath, Pilot contamination and precoding in multi-cell TDD systems. IEEE Trans. Wireless Commun. 10(8), 2640-2651 (2011)

10. N Krishnan, RD Yates, NB Mandayam, Uplink linear receivers for multi-cell multiuser mimo with pilot contamination: large system analysis. IEEE Trans. Wireless Commun. 13(8), 4360-4373 (2014)

11. A Duly, T Kim, D Love, J Krogmeier, Closed-loop beam alignment for massive MIMO channel estimation. IEEE Commun. Lett. 18(8), 1439-1442 (2014)

12. A Adhikary, J Nam, J Ahn, G Caire, Joint spatial division and multiplexing-the large-scale array regime. IEEE Trans. Inf. Theory. 59(10) 6441-6463 (2013)

13. C Sun, X Gao, S Jin, M Matthaiou, Z Ding, C Xiao, Beam division multiple access transmission for massive mimo communications. IEEE Trans. Commun. 63(6), 2170-2184 (2015)

14. D Ying, FW Vook, TA Thomas, DJ Love, A Ghosh, in IEEE International Conference on Commun. (ICC). Kronecker product correlation model and limited feedback codebook design in a 3D channel model (IEEE, Sydney, 2014), pp. 5865-5870

15. Z Wang, W Liu, C Qian, S Chen, L Hanzo, Two-dimensional precoding for 3-d massive mimo. IEEE Trans. Veh. Technol. 66(6), 5485-5490 (2017)

16. X Li, S Jin, X Gao, RW Heath, Three-dimensional beamforming for large-scale fd-mimo systems exploiting statistical channel state Information. IEEE Trans. Veh. Technol. 65(11), 8992-9005 (2016)

17. X Li, S Jin, HA Suraweera, J Hou, X Gao, Statistical 3-d beamforming for large-scale mimo downlink systems over rician fading channels. IEEE Trans. Commun. 64(4), 1529-1543 (2016)
18. Z Zhang, X Chai, K Long, AV Vasilakos, L Hanzo, Full duplex techniques for $5 G$ networks: self-interference cancellation, protocol design, and relay selection. IEEE Commun. Mag. 53(5), 128-137 (2015)

19. D Kim, H Lee, D Hong, A survey of in-band full-duplex transmission: from the perspective of PHY and MAC layers. IEEE Commun. Surv. Tutorials. 17(4), 2017-2046 (2015)

20. A Sabharwal, P Schniter, D Guo, DW Bliss, S Rangarajan, R Wichman, In-band full-duplex wireless: challenges and opportunities. IEEE J. Sel. Areas Commun. 32(9), 1637-1652 (2014)

21. C Psomas, M Mohammadi, I Krikidis, HA Suraweera, Impact of directionality on interference mitigation in full-duplex cellular networks. IEEE Trans. Wireless Commun. 16(1), 487-502 (2017)

22. M Mohammadi, HA Suraweera, Y Cao, I Krikidis, C Tellambura, Full-duplex radio for uplink/downlink wireless access with spatially random nodes. IEEE Trans. Commun. 63(12), 5250-5266 (2015)

23. J Lee, TQS Quek, Hybrid full-/half-duplex system analysis in heterogeneous wireless networks. IEEE Trans. Wirel. Commun. 14(5), 2883-2895 (2015)

24. HQ Ngo, HA Suraweera, M Matthaiou, EG Larsson, Multipair full-duplex relaying with massive arrays and linear processing. IEEE J. Sel. Areas Commun. 32(9), 1721-1737 (2014)

25. XXia, D Zhang, K Xu, W Ma, Y Xu, Hardware Impairments Aware Transceiver for Full-Duplex Massive MIMO Relaying. IEEE Trans. Signal Process. 63(24), 6565-6580 (2015)

26. M Mohammadi, BK Chalise, HA Suraweera, Z Ding, in IEEE International Conference on Communications. Wireless information and power transfer in full-duplex systems with massive antenna arrays (IEEE, Paris, 2017), pp. 1-6

27. Y Li, P Fan, A Leukhin, L Liu, On the Spectral and energy efficiency of full-duplex small-cell wireless systems with massive mimo. IEEE Trans. Veh. Technol. 66(3), 2339-2353 (2017)

28. XXia, Y Xu, KXu, D Zhang, W Ma, Full-duplex massive mimo AF relaying with semiblind gain control. IEEE Trans. Veh. Technol. 65(7), 5797-5804 (2016)

29. H Yin, D Gesbert, M Filippou, Y Liu, A coordinated approach to channel estimation in large-scale multiple-antenna systems. IEEE J. Sel. Areas Commun. 31(2), 264-273 (2013)

30. JP Kermoal, L Schumacher, KI Pedersen, PE Mogensen, F Frederiksen, A stochastic MIMO radio channel model with experimental validation. IEEE J. Sel. Areas Commun. 20(6), 1211-1226 (2002)

31. B Clerckx, C Oestges, MIMO Wireless Networks: Channels, Techniques and Standards for Multi-Antenna, Multi-User and Multi-Cell Systems. 2nd. (Academic Press, Oxford, 2013)

32. J Wang, R Zhang, W Duan, SX Lu, L Cai, in Proc. ICC 2014. Angular spread measurement and modeling for $3 \mathrm{D} \mathrm{MIMO}$ in urban macrocellular radio channels (IEEE, Sydney, 2014), pp. 20-25

33. MA Maddah-Ali, DNC Tse, Completely stale transmitter channel state information is still very useful. IEEE Trans. Inform. Theory. 58(7), 4418-4432 (2012)

34. T Kailath, AH Sayed, B Hassibi, Linear Estimation. (Upper Saddle River, Prentice-Hall, 2000)

35. B Hassibi, BM Hochwald, How much training is needed in multiple-antenna wireless links? IEEE Trans. Inf. Theory. 49(4), 951-963 (2003)

36. 3GPP TR 36.828, Further enhancements to LTE time division duplex (TDD) for downlink-uplink (DL-UL) interference management and traffic adaptation. (2012). v.11.0.0. www.3gpp.org. Accessed 7 Aug 2018

37. Y Song, S Nagata, H Jiang, L Chen, in IEEE International Conference on Communications. CSI-RS design for 3D MIMO in future LTE-Advanced (IEEE, Sydney, 2014), pp. 5101-5106

38. G Caire, N Jindal, M Kobayashi, N Ravindran, Multiuser MIMO achievable rates with downlink training and channel state feedback. IEEE Trans. Inf. Theory. 56(6), 2845-2866 (2010)

39. J Choi, D Love, T Kim, Trellis-extended codebooks and successive phase adjustment: A path from LTE-Advanced to FDD massive MIMO systems. IEEE Trans. Wirel. Commun. 14(4), 2007-2016 (2015) 